\title{
The amplitude for classical gravitational scattering at third Post-Minkowskian order
}

\author{
N. Emil J. Bjerrum-Bohr, ${ }^{a}$ Poul H. Damgaard, ${ }^{a, d}$ Ludovic Planté $^{a}$ \\ and Pierre Vanhove ${ }^{b, c, d}$ \\ ${ }^{a}$ Niels Bohr International Academy, Niels Bohr Institute, University of Copenhagen, \\ Blegdamsvej 17, DK-2100 Copenhagen, Denmark \\ ${ }^{b}$ Institut de Physique Theorique, Université Paris-Saclay, \\ CEA, CNRS, F-91191 Gif-sur-Yvette Cedex, France \\ ${ }^{c}$ National Research University Higher School of Economics, \\ Russian Federation \\ ${ }^{d}$ Theoretical Physics Department, CERN, \\ 1211 Geneva 23, Switzerland \\ E-mail: bjbohr@nbi.dk, phdamg@nbi.dk, pnm654@nbi.ku.dk, \\ pierre.vanhove@ipht.fr
}

ABSTRACT: We compute the scattering amplitude for classical black-hole scattering to third order in the Post-Minkowskian expansion, keeping all terms needed to derive the scattering angle to that order from the eikonal formalism. Our results confirm a conjectured relation between the real and imaginary parts of the amplitude by Di Vecchia, Heissenberg, Russo, and Veneziano, and are in agreement with a recent computation by Damour based on radiation reaction in general relativity.

KEywords: Scattering Amplitudes, Classical Theories of Gravity, Effective Field Theories ArXiv EPrint: 2105.05218 


\section{Contents}

1 Introduction 1

2 Einstein gravity at two-loop order 3

3 Contributions from the three-graviton cut 4

3.1 Double-box contributions 5

$\begin{array}{lll}3.2 & \text { Box-triangle contributions } & 7\end{array}$

3.3 Double-triangle contributions 8

3.4 The $H$-diagram 9

$\begin{array}{ll}3.5 & \text { Box-bubble contribution }\end{array}$

4 Self-energy diagrams and vertex corrections $\quad 9$

5 The two-loop amplitude and the eikonal phase 11

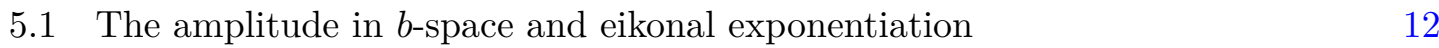

$\begin{array}{ll}5.2 & \text { Relation to the world-line formalism: velocity cuts } \\ 5.3\end{array}$

$\begin{array}{lll}5.3 & \text { The scattering angle } & 18\end{array}$

6 Conclusion 20

A The five-point tree amplitude and the three-graviton cut 21

B Numerator factors $\quad 22$

C The one-loop two-body amplitude $\quad 24$

$\begin{array}{ll}\text { D Master integrals } & 27\end{array}$

\section{Introduction}

The Post-Minkowskian expansion of Einstein gravity is arguably one of the most fertile areas in which to apply modern methods of amplitude calculations [1-4]. Being based solely on an expansion in Newton's constant $G_{N}$, results are valid at all velocities and are thus effectively re-summing an infinite number of terms of the perhaps more familiar Post-Newtonian expansion. Progress has been very rapid and first results for the conservative part of the interaction Hamiltonian are now known to both third and fourth order in the Post-Minkowskian expansion [5-8]. These computations are firmly rooted in established quantum field theoretic methods, be they phrased in terms of effective field theory matching [4] or, equivalently, in terms of the standard definition of a two-body interaction potential from the iterated solution of the Lippmann-Schwinger equation, which quantify exactly what is commonly known as Born subtractions [9]. The conservative part of the scattering to third Post-Minkowskian has also been confirmed in the framework of the world-line approach [10]. A remarkable relationship between the relativistic 
two-body kinematics and the iterated solution to the Lippmann-Schwinger equation leads to explicit formulas for the scattering angle in terms of the coefficients of the relativistic two-body potential [11-13], in a sense thereby solving the problem of kinematics of the relativistic two-body problem in general relativity by means of quantum field theory and amplitude techniques.

The puzzling aspect of an ill-defined high-energy limit of the conservative part of the two-body interaction Hamiltonian at third Post-Minkowskian order [6] has recently led to surprising new insight into the relationship between the two-to-two gravitational scattering amplitude and general relativity [14-17]. From the amplitude point of view, refs. [14, 16, 17] have shown how certain classical terms of the two-loop scattering, that are not captured by a limitation to the so-called potential region of the loop integrals, restore a well-behaved high-energy behavior. This picture has been understood directly from general relativity in terms of gravitational radiation reaction [15]. Indeed, in the low-energy limit the new terms give rise to half-integer powers in the Post-Newtonian expansion and thus belong to effects described by radiation. By using the formalism of ref. [18] a direct computation of the radiated momentum to that order leads to the same result for the scattering angle [19, 20] (see also [21]).

A natural language for the calculation of the scattering angle of two black holes in general relativity is the gravitational eikonal. It relies on the exponentiation of appropriate terms of the $S$-matrix in the small-angle limit of the involved semi-classical field theory amplitudes. Exponentiation has been proven to all orders for Einstein gravity at both leading Post-Minkowskian counting [22] and next-to-leading order for equal masses [23] (generalized to different masses in ref. [3]). This powerful framework has led to the famous prediction for high-energy gravitational scattering by Amati, Ciafaloni, and Veneziano [24] and has recently been explored and extended in numerous directions [14, 16, 17, 25-31]. Although the eikonal formalism is used to derive the classical scattering angle, an interesting feature is that also an in principle infinite number of super-classical terms (corresponding to inverse powers of $\hbar$ ) must be computed as well in order to confirm the exponentiation of the amplitude in impact parameter space, a phenomenon that ultimately must follow from unitarity alone [31]. Moreover, an intricate interplay between classical and quantum pieces of the amplitude conspire to provide the correct classical scattering angle at any given order in the Post-Minkowskian expansion.

Crucial to the argument of refs. $[14,16,17]$ is a remarkably simple relation between the divergent part of the imaginary part of the amplitude and the finite real part of the radiation-reaction contribution. In maximal supergravity, this relation has recently been explicitly confirmed from the two-to-two scattering amplitude [32] as well as from the radiated momentum calculation [20]. Extended to general relativity, the entirely different approach of ref. [15] should leave little doubt that this relation holds in Einstein gravity as well. Nevertheless, an explicit confirmation from a full amplitude calculation seems needed at this stage. The purpose of the present paper is to fill this gap and, hopefully, provide some further insight into the result. We shall employ the method recently described in ref. [32]. The idea is to organize the integrand of loop amplitudes in subsets that naturally, due to the $i \varepsilon$-prescription of the Feynman propagator, combine into delta functions over 
momenta in a manner reminiscent of eikonal calculations [22] and which have earlier also been used to simplify the evaluation of some of the involved loop integrals [6,21]. Imposing these delta functions lowers the dimensionality of the integrals in a covariant manner. A second advantage of the method of ref. [32] is that it significantly reduces the number of master integrals that need to be known. This was obvious in the case of maximal supergravity and, as we shall demonstrate in this paper, this holds in Einstein gravity as well. No new master integrals are needed as compared to the supergravity case [32]. In the end, we believe that we here for the first time compute all terms of the gravitational scattering amplitude of massive objects that are needed to obtain the classical scattering angle to third Post-Minkowskian order. Simultaneously, we hope that this can help paving the way for more efficient evaluations at even higher orders. We also explicitly confirm the relation between the divergent imaginary part and the real part of the radiation-reaction piece that was put forward in refs. [14, 16, 17].

\section{Einstein gravity at two-loop order}

Our starting point is the Einstein-Hilbert Lagrangian minimally coupled to two massive scalar fields:

$$
\mathcal{L}_{\mathrm{EH}}=\int d^{4} x \sqrt{-g}\left[\frac{R}{16 \pi G_{N}}+\frac{1}{2} g^{\mu \nu}\left(\partial_{\mu} \phi_{1} \partial_{\nu} \phi_{1}+\partial_{\mu} \phi_{2} \partial_{\nu} \phi_{2}\right)-\frac{m_{1}^{2}}{2} \phi_{1}^{2}-\frac{m_{2}^{2}}{2} \phi_{2}^{2}\right] .
$$

Here, $R$ defines the Ricci scalar and $g$ is the determinant of the metric: $g_{\mu \nu}(x) \equiv \eta_{\mu \nu}+$ $\sqrt{32 \pi G_{N}} h_{\mu \nu}(x)$ expanded around a Minkowski background, diag $\eta_{\mu \nu} \equiv(1,-1,-1,-1)$.

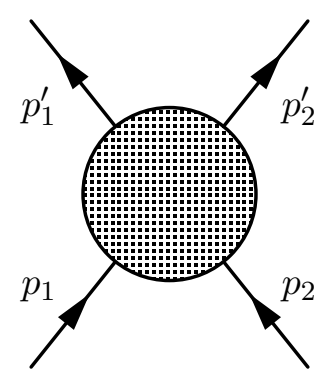

We consider here the two-to-two amplitude with $p_{1}$ and $p_{2}$ denoting incoming momenta and $p_{1}^{\prime}$ and $p_{2}^{\prime}$ outgoing momenta such that $p_{1}^{2}={p_{1}^{\prime}}^{2}=m_{1}^{2}$ and $p_{2}^{2}=p_{2}^{\prime 2}=m_{2}^{2}$.

We work in the center of mass frame and our conventions for the kinematical invariants are:

$$
\begin{aligned}
& s \equiv\left(p_{1}+p_{2}\right)^{2}=\left(p_{1}^{\prime}+p_{2}^{\prime}\right)^{2}=m_{1}^{2}+m_{2}^{2}+2 m_{1} m_{2} \sigma, \quad \sigma \equiv \frac{p_{1} \cdot p_{2}}{m_{1} m_{2}} \\
& t \equiv\left(p_{1}-p_{1}^{\prime}\right)^{2}=\left(p_{2}^{\prime}-p_{2}\right)^{2} \equiv q^{2}=-\vec{q}^{2}
\end{aligned}
$$

and

$$
u \equiv\left(p_{1}-p_{2}^{\prime}\right)^{2}=\left(p_{1}^{\prime}-p_{2}\right)^{2},
$$

so that, as usual, $s$ gives the center of mass energy, $E_{C M}^{2}$, and $t$ is the transferred momentum. The classical limit is extracted by sending $\hbar \rightarrow 0$ and keeping $\underline{q}=q / \hbar$ fixed. 
The two-loop amplitude we evaluate below is composed by a piece coming from the three-particle cut with only gravitons propagating across the cut

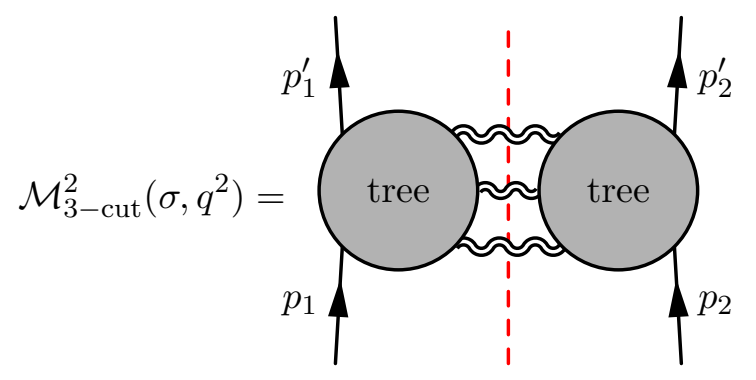

and another piece from the self-energy and vertex correction contributions given in section 4

$$
\mathcal{M}_{2}\left(\sigma, q^{2}\right)=\mathcal{M}_{2}^{3-\text { cut }}\left(\sigma, q^{2}\right)+\mathcal{M}_{2}^{\text {self-energy }}\left(\sigma, q^{2}\right) .
$$

The three particle-cut is defined in $D=4-2 \epsilon$ dimensions as

$$
\begin{aligned}
\mathcal{M}_{2}^{3-\text { cut }}\left(\sigma, q^{2}\right) & =\int \frac{d^{D} l_{1} d^{D} l_{2} d^{D} l_{3}}{(2 \pi)^{3 D}}(2 \pi)^{D} \delta^{(D)}\left(l_{1}+l_{2}+l_{3}+q\right) \frac{i^{3}}{l_{1}^{2} l_{2}^{2} l_{3}^{2}} \\
\times & \frac{1}{3 !} \sum_{\substack{\text { Perm }\left(l_{1}, l_{2}, l_{3}\right) \\
\lambda_{1}= \pm, \lambda_{2}= \pm, \lambda_{3}= \pm}} \mathcal{M}_{0}\left(p_{1}, p_{1}^{\prime}, l_{1}^{\lambda_{1}}, l_{2}^{\lambda_{2}}, l_{3}^{\lambda_{3}}\right)\left(\mathcal{M}_{0}\left(p_{2}, p_{2}^{\prime},-l_{1}^{\lambda_{1}},-l_{2}^{\lambda_{2}},-l_{3}^{\lambda_{3}}\right)\right)^{*}
\end{aligned}
$$

which involves the five points tree-level amplitudes given in the appendix A. The sum is over the helicity configuration $\lambda_{i}$ of the gravitons across the cut. We only keep the cut-constructible part of the two-loop amplitude.

The three-particle cut gives both the conservative part of the classical potential plus some of the radiation-reaction pieces. A few self-energy and vertex corrrection diagrams are needed to get the full radiation-reaction term. Based on the $\hbar$ counting in eq. (3.6) of [32] it is clear the one needs a least two massive propagators to get a classical contribution to the amplitude. By inspection of the possible topology of the Feynman graphs we conclude that the three-particle cut contribution, the self-energy and vertex corrrection diagrams are the only one contributing to this order.

\section{Contributions from the three-graviton cut}

The important difference with the maximal supergravity computation in [32] is that the two-loop amplitude involves integrals with non-trivial numerators and with more topologies. Using a partial fraction decomposition of the tree-level amplitudes with respects to the linear propagators $p_{1} \cdot l_{i}$ and $p_{2} \cdot l_{i}$ with $i=1,2,3$, one can reorganise the three-particle cut into five distinct topologies that will contribute to the classical result

$$
\mathcal{M}_{2}^{3-\text { cut }}\left(\sigma, q^{2}\right)=\mathcal{M}_{2}^{\square}+\mathcal{M}_{2}^{\triangleleft}+\mathcal{M}_{2}^{\square}+\mathcal{M}_{2}^{\triangleleft \triangleleft}+\mathcal{M}_{2}^{\triangleright \triangleright}+\mathcal{M}_{2}^{H}+\mathcal{M}_{2}^{\square \circ}
$$



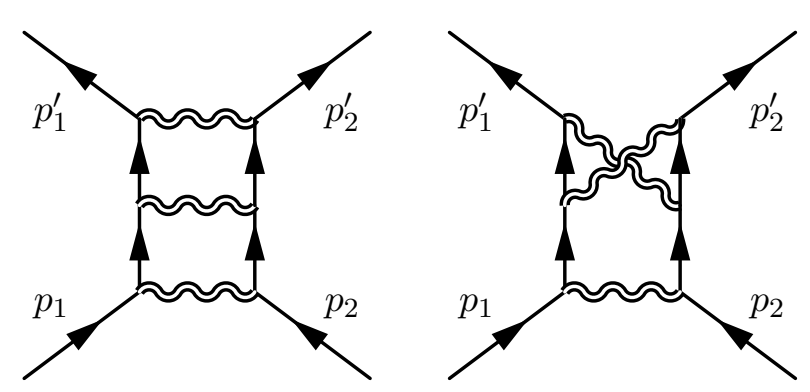

Figure 1. Double-box and crossed double-box diagrams.

with the result

$$
\begin{gathered}
\mathcal{M}_{2}^{3-\operatorname{cut}(-1)}\left(\sigma, q^{2}\right)=\frac{2\left(4 \pi e^{-\gamma_{E}}\right)^{2 \epsilon} \pi G_{N}^{3} m_{1}^{2} m_{2}^{2}}{3 \epsilon|\underline{q}|^{4 \epsilon} \hbar}\left(\frac{3 s\left(2 \sigma^{2}-1\right)^{3}}{\left(\sigma^{2}-1\right)^{2}}\right. \\
+\frac{i m_{1} m_{2}\left(2 \sigma^{2}-1\right)}{\pi \epsilon\left(\sigma^{2}-1\right)^{\frac{3}{2}}}\left(\frac{1-49 \sigma^{2}+18 \sigma^{4}}{5}-\frac{6 \sigma\left(2 \sigma^{2}-1\right)\left(6 \sigma^{2}-7\right) \operatorname{arccosh}(\sigma)}{\sqrt{\sigma^{2}-1}}\right) \\
-\frac{9\left(2 \sigma^{2}-1\right)\left(1-5 \sigma^{2}\right) s}{2\left(\sigma^{2}-1\right)}+\frac{3}{2}\left(m_{1}^{2}+m_{2}^{2}\right)\left(-1+18 \sigma^{2}\right)-m_{1} m_{2} \sigma\left(103+2 \sigma^{2}\right) \\
+\frac{12 m_{1} m_{2}\left(3+12 \sigma^{2}-4 \sigma^{4}\right) \operatorname{arccosh}(\sigma)}{\sqrt{\sigma^{2}-1}} \\
\left.-\frac{6 i m_{1} m_{2}\left(2 \sigma^{2}-1\right)^{2}}{\pi \epsilon \sqrt{\sigma^{2}-1}}\left(\frac{-1}{4\left(\sigma^{2}-1\right)}\right)^{\epsilon} \frac{d}{d \sigma}\left(\frac{\left(2 \sigma^{2}-1\right) \operatorname{arccosh}(\sigma)}{\sqrt{\sigma^{2}-1}}\right)\right) .
\end{gathered}
$$

The expressions for the numerator factors of each of these integrals are given in appendix B. The double-box integrals $\mathcal{M}_{2}^{\square}$ are evaluated in section 3.1, the box-triangles $\mathcal{M}_{2}^{\triangleleft}$ and $\mathcal{M}_{2}^{\square}$ are evaluated in section 3.2, the double-triangle integrals $\mathcal{M}_{2}^{\triangleright \triangleright}$ and $\mathcal{M}_{2}^{\triangleleft \triangleleft}$ are evaluated in section 3.3, the $H$-diagram contributions $\mathcal{M}_{2}^{H}$ are evaluated in section 3.4, and the box-bubble contributions $\mathcal{M}_{2}^{\square \circ}$ are evaluated in section 3.5.

\subsection{Double-box contributions}

The double-box contributions arise from the sum of Feynman graph topologies given in figure 1. We provide the numerator factors in appendix B. Performing the tensorial reductions with LiteRed [33], we find that the double-box contribution has the expansion

$$
\begin{array}{r}
\mathcal{M}_{2}^{\square}\left(\sigma, q^{2}\right)=4096 \pi^{3} G_{N}^{3} m_{1}^{5} m_{2}^{5}\left(2 \sigma^{2}-1\right)^{2}\left(m_{1} m_{2}\left(2 \sigma^{2}-1\right)\left(J_{s}+J_{u}\right)-6 \sigma \hbar^{2}|\underline{q}|^{2} J_{u}\right. \\
\left.+8 \sigma \hbar^{2}|q|^{2} J_{\square}^{N P}\right) .
\end{array}
$$

The sum of the integrals $J_{s}+J_{u}$ has been evaluated in section 4.3 of [32], and the integral $J_{u}$ in section 4.2 of [32]. 
Evaluation of the integral $\boldsymbol{J}_{\square}^{N P}$. This is a new contribution that did not appear in the maximal supergravity computation of ref. [32]. It reads (with $D=4-2 \epsilon$ )

$$
\begin{aligned}
J_{\square}^{N P}=\frac{|q|^{2 D-10}}{16 \hbar^{3}} & \int \frac{d^{D} l_{1} d^{D} l_{2}}{(2 \pi)^{2 D}} \frac{l_{2} \cdot l_{3}}{l_{1}^{2} l_{2}^{2}\left(l_{1}+l_{2}+u_{q}\right)^{2}} \\
\times & \left(\frac{1}{\left(\bar{p}_{1} \cdot l_{1}+i \varepsilon\right)\left(\bar{p}_{1} \cdot l_{2}-i \varepsilon\right)}-\frac{1}{\left(\bar{p}_{1} \cdot l_{2}+i \varepsilon\right)\left(\bar{p}_{1} \cdot l_{1}-i \varepsilon\right)}\right) \\
& \quad \times\left(\frac{1}{\left(\bar{p}_{2} \cdot l_{1}-i \varepsilon\right)\left(\bar{p}_{2} \cdot l_{3}+i \varepsilon\right)}-\frac{1}{\left(\bar{p}_{2} \cdot l_{3}-i \varepsilon\right)\left(\bar{p}_{2} \cdot l_{1}+i \varepsilon\right)}\right),
\end{aligned}
$$

where we have scaled the loop momenta $l_{i} \rightarrow \hbar l_{i} \underline{q}$ as in [32], and defined $p_{1}=\bar{p}_{1}+\frac{\hbar q}{2}$, $p_{2}=\bar{p}_{2}-\frac{\hbar \underline{q}}{2}$ and $q=|\vec{q}| u_{q}$ with $u_{q}^{2}=-1$.

Using the definition of the delta-function as a distribution

$$
\lim _{\varepsilon \rightarrow 0^{+}}\left(\frac{1}{x-i \varepsilon}-\frac{1}{x+i \varepsilon}\right)=\lim _{\varepsilon \rightarrow 0^{+}} \frac{2 i \varepsilon}{x^{2}+\varepsilon^{2}}=2 i \pi \delta(x),
$$

the above expression can be written in terms of delta functions:

$$
\begin{aligned}
J_{\square}^{N P}= & -\frac{|q|^{2 D-10}}{16 \hbar^{3}} \int \frac{d^{D} l_{1} d^{D} l_{2}}{(2 \pi)^{2 D-2}} \frac{l_{2} \cdot l_{3}}{l_{1}^{2} l_{2}^{2}\left(l_{1}+l_{2}+u_{q}\right)^{2}}\left(\frac{\delta\left(\bar{p}_{1} \cdot l_{1}\right)}{\bar{p}_{1} \cdot l_{2}+i \varepsilon}-\frac{\delta\left(\bar{p}_{1} \cdot l_{2}\right)}{\bar{p}_{1} \cdot l_{1}+i \varepsilon}\right) \\
& \times\left(\frac{\delta\left(\bar{p}_{2} \cdot l_{3}\right)}{\bar{p}_{2} \cdot l_{1}+i \varepsilon}-\frac{\delta\left(\bar{p}_{2} \cdot l_{1}\right)}{\bar{p}_{2} \cdot l_{3}+i \varepsilon}\right) .
\end{aligned}
$$

We can therefore express the sum of all the double-box terms in Einstein gravity in terms of integrals with delta-functions exactly as in the corresponding computation for maximal supergravity [20,32].

Using LiteRed [33] we expand this expression on the master integrals used in [32] (see appendix D for a summary of the results) ${ }^{1}$

$$
\begin{aligned}
J_{\amalg}^{N P}= & \frac{|\underline{q}|^{2 D-10}}{96 \hbar^{3} \epsilon^{4} m_{1}^{2} m_{2}^{2}\left(\sigma^{2}-1\right)}\left(\mathcal{I}_{4}(\sigma)+2 \mathcal{I}_{9}(\sigma)\right)-\frac{|\underline{q}|^{2 D-10}}{96 \hbar^{3} \epsilon^{4} m_{1}^{2} m_{2}^{2}\left(\sigma^{2}-1\right)}\left(\mathcal{I}_{4}(\sigma)-\mathcal{I}_{9}(\sigma)\right) \\
& -\frac{|q|^{2 D-10}}{32 \hbar^{3}} \frac{4(\operatorname{arccosh}(\sigma)-i \pi)}{m_{1} m_{2} \sqrt{\sigma^{2}-1}} \frac{i\left(4 \pi e^{-\gamma_{E}}\right)^{2 \epsilon}}{128 m_{1} m_{2} \epsilon^{2} \pi^{3} \sqrt{\sigma^{2}-1}} .
\end{aligned}
$$

Next, using the evaluation of the master integral $\mathcal{I}_{4}(\sigma)$ in section 5.2 and $\mathcal{I}_{9}(\sigma)$ in section 5.7 of ref. [32] we have

$$
J_{\square}^{N P}=\frac{|q|^{2 D-10}\left(4 \pi e^{-\gamma_{E}}\right)^{2 \epsilon}}{1024 \hbar^{3} \epsilon^{2} \pi^{2} m_{1}^{2} m_{2}^{2}\left(\sigma^{2}-1\right)}\left(\frac{i}{\pi}\left(\frac{-1}{4}\right)^{\epsilon} \int_{1}^{\sigma} \frac{d t}{\left(t^{2}-1\right)^{\frac{1}{2}+\epsilon}}-\frac{i}{\pi} \operatorname{arccosh}(\sigma)\right) .
$$

The double-box. Summing everything, the double-box contribution is given by

$$
\mathcal{M}_{2}^{\square}\left(\sigma, q^{2}\right)=\frac{1}{\mid \underline{q}^{\mid \epsilon}}\left(\mathcal{M}_{2}^{\square(-3)}\left(\sigma, q^{2}\right)+\mathcal{M}_{2}^{\square(-2)}\left(\sigma, q^{2}\right)+\mathcal{M}_{2}^{\square(-1)}\left(\sigma, q^{2}\right)+\mathcal{O}(\hbar)\right),
$$

${ }^{1}$ The tensorial reduction is done after having localised the integrals with the delta-function insertions. The tensorial reduction is then performed on the $D-1$ dimensional integrals. 
where the superscript indicates the order of $\hbar$, with the leading term

$$
\mathcal{M}_{2}^{\square(-3)}=-\frac{128 \pi^{3} G_{N}^{3} m_{1}^{4} m_{2}^{4}\left(2 \sigma^{2}-1\right)^{3} \Gamma(-\epsilon)^{3} \Gamma(1+2 \epsilon)}{3 \hbar^{3}|\underline{q}|^{2}\left(\sigma^{2}-1\right)(4 \pi)^{2-2 \epsilon} \Gamma(-3 \epsilon)} .
$$

The first sub-leading piece is

$$
\mathcal{M}_{2}^{\square(-2)}=\frac{256 \pi^{3} G_{N}^{3} i m_{1}^{3} m_{2}^{3}\left(m_{1}+m_{2}\right)\left(2 \sigma^{2}-1\right)^{3}}{\hbar^{2}|\underline{q}|\left(\sigma^{2}-1\right)^{\frac{3}{2}}} \frac{\Gamma\left(\frac{1}{2}-\epsilon\right)^{2} \Gamma\left(\frac{1}{2}+2 \epsilon\right) \Gamma(-\epsilon) \Gamma\left(\frac{1}{2}-2 \epsilon\right)}{(4 \pi)^{\frac{5}{2}-2 \epsilon} \Gamma\left(\frac{1}{2}-3 \epsilon\right) \Gamma(-2 \epsilon)},
$$

and the classical term, when written at leading orders in $\epsilon,{ }^{2}$ is

$$
\begin{aligned}
\mathcal{M}_{2}^{\square(-1)}=\frac{4 G_{N}^{3} m_{1}^{3} m_{2}^{3}\left(2 \sigma^{2}-1\right)^{2}}{\hbar \pi}\left(4 \pi e^{-\gamma_{E}}\right)^{2 \epsilon}\left(\frac{\pi^{2} s\left(2 \sigma^{2}-1\right)}{2 \epsilon m_{1} m_{2}\left(\sigma^{2}-1\right)^{2}}\right. & \frac{i \pi\left(\left(7-6 \sigma^{2}\right) \sigma \operatorname{arccosh}(\sigma)-\left(2 \sigma^{2}-1\right) \sqrt{\sigma^{2}-1}\right)}{\epsilon^{2}\left(\sigma^{2}-1\right)^{2}} \\
+ & \left.\frac{i \pi}{\epsilon^{2} \sqrt{\sigma^{2}-1}}\left(\frac{-1}{4\left(\sigma^{2}-1\right)}\right)^{\epsilon} \frac{d}{d \sigma}\left(\frac{\left(2 \sigma^{2}-1\right) \operatorname{arccosh}(\sigma)}{\sqrt{\sigma^{2}-1}}\right)\right) .
\end{aligned}
$$

The first and the second lines agree with first quantum correction to the one-loop box as given in ref. [27]. These pieces are needed for the exponentiation of the eikonal phase to two-loop order. The last line is the radiation-reaction term. We have written it compactly in terms of a $\sigma$-derivative. Notice that in Einstein gravity this term is

$$
\left(2 \sigma^{2}-1\right)^{2} \frac{d}{d \sigma}\left(\frac{\left(2 \sigma^{2}-1\right) \operatorname{arccosh}(\sigma)}{\sqrt{\sigma^{2}-1}}\right)
$$

whereas it is

$$
\left(2 \sigma^{2}\right)^{2} \frac{d}{d \sigma}\left(\frac{2 \sigma^{2} \operatorname{arccosh}(\sigma)}{\sqrt{\sigma^{2}-1}}\right)
$$

in maximal supergravity. The difference is due to the exchange of the dilaton field in the latter theory.

\subsection{Box-triangle contributions}

The box-triangle contributions are given by the sum of Feynman integrals topologies given in figure 2, together with the mirrored ones with the graviton line attached to the other scalar line. The numerator factors are again provided in appendix B. Using LiteRed [33] for performing the tensorial reduction, and after evaluation of the various integrals, this contribution expands into

$$
\mathcal{M}_{2}^{\square}=\frac{1}{\mid \underline{q}^{4 \epsilon}}\left(\mathcal{M}_{2}^{\square(-2)}+\mathcal{M}_{2}^{\square(-1)}+\mathcal{O}\left(\hbar^{0}\right)\right) .
$$

\footnotetext{
${ }^{2}$ For certain terms we keep $\epsilon$ in the exponent for reasons that will become clear below.
} 

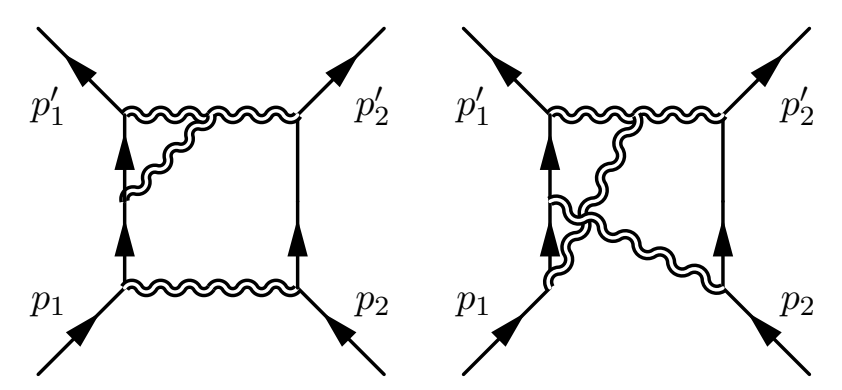

Figure 2. Box-triangle graphs.
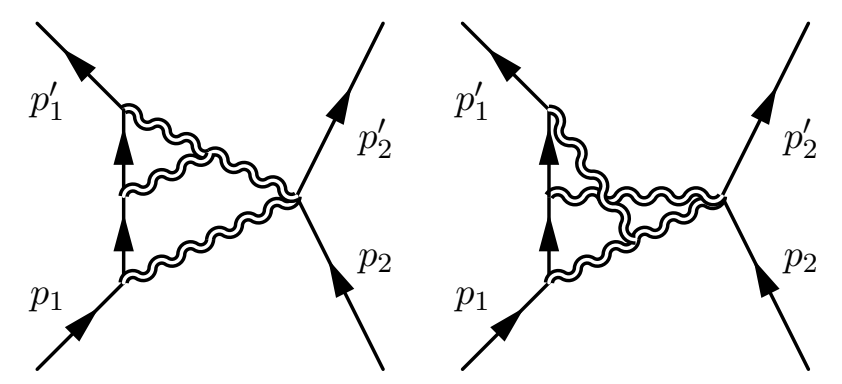

Figure 3. Double-triangle graphs.

There is no contribution of order $1 / \hbar^{3}$. The leading order in the $\hbar$ expansion is

$$
\mathcal{M}_{2}^{\square(-2)}=\frac{i 6 \pi^{2} G_{N}^{3} m_{1}^{4} m_{2}^{3}\left(2 \sigma^{2}-1\right)\left(1-5 \sigma^{2}\right)\left(4 \pi e^{-\gamma_{E}}\right)^{2 \epsilon}}{\epsilon \sqrt{\sigma^{2}-1} \hbar^{2}|q|}+\mathcal{O}\left(\epsilon^{0}\right),
$$

and the classical term is

$$
\begin{aligned}
\mathcal{M}_{2}^{\square(-1)}= & \left(4 \pi e^{-\gamma_{E}}\right)^{2 \epsilon} \frac{128 \pi^{3} G_{N}^{3} m_{1}^{3} m_{2}^{2}}{3 \hbar}\left(\frac{3 i m_{2}\left(2 \sigma^{2}-1\right)\left(22 \sigma^{2}-1\right)}{64 \epsilon^{2} \pi^{3} \sqrt{\sigma^{2}-1}}\right. \\
& \left.-\frac{9\left(2 \sigma^{2}-1\right)\left(1-5 \sigma^{2}\right)\left(m_{1}+m_{2} \sigma\right)}{128\left(\sigma^{2}-1\right) \epsilon \pi^{2}}-\frac{3\left(2 \sigma^{2}-1\right) m_{1}}{128 \epsilon \pi^{2}}-\frac{m_{2} \sigma\left(55+2 \sigma^{2}\right)}{128 \epsilon \pi^{2}}\right) .
\end{aligned}
$$

The symmetric contribution $\mathcal{M}_{2}^{\triangleleft}$ is simply obtained by the exchange of $m_{1} \leftrightarrow m_{2}$.

\subsection{Double-triangle contributions}

The double-triangle contributions are given by the sum of Feynman integrals topologies given in figure 3 together with the mirrored ones with the triangle attached to the other scalar line. We give the numerator factors in appendix B.

We again use LiteRed [33] for the tensorial reduction. Applying also the identity in (3.5) we obtain the delta-function representation

$$
\begin{aligned}
\mathcal{M}_{2}^{\triangleright \triangleright} & =-\frac{128 \pi^{3} G_{N}^{3} m_{1}^{6} m_{2}^{2}\left(10 \sigma^{2}-1\right)}{\hbar|\underline{q}|^{4 \epsilon}} \int \frac{d^{D-1} l_{1} d^{D-1} l_{2}}{(2 \pi)^{2 D-2}} \frac{\delta\left(\bar{p}_{1} \cdot l_{1}\right) \delta\left(\bar{p}_{1} \cdot l_{2}\right)}{l_{1}^{2} l_{2}^{2}\left(l_{1}+l_{2}+u_{q}\right)^{2}}, \\
& =\frac{2 \pi G_{N}^{3} m_{1}^{4} m_{2}^{2}\left(10 \sigma^{2}-1\right)\left(4 \pi e^{-\gamma_{E}}\right)^{2 \epsilon}}{\epsilon|\underline{q}|^{4 \epsilon} \hbar},
\end{aligned}
$$




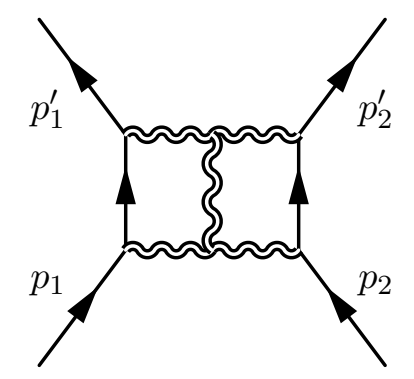

Figure 4. The $H$ diagram.

and the symmetric triangle

$$
\mathcal{M}_{2}^{\triangleleft \triangleleft}=\frac{2 \pi G_{N}^{3}|\underline{q}|^{-4 \epsilon} m_{1}^{2} m_{2}^{4}\left(10 \sigma^{2}-1\right)\left(4 \pi e^{-\gamma_{E}}\right)^{2 \epsilon}}{\epsilon|\underline{q}|^{4 \epsilon} \hbar} .
$$

Notice that the double-triangle integrals start contributing from the classical order in the $\hbar \rightarrow 0$ limit.

\subsection{The $\boldsymbol{H}$-diagram}

The $H$-diagram integral is the $t$-channel integral similar to the one evaluated in section 4.1 of [32] but this time with tensorial numerator factor. Reducing the numerator using LiteRed [33] we get the expression

$$
\mathcal{M}_{2}^{H}=-\frac{256 \pi^{3} G_{N}^{3} m_{1}^{3} m_{2}^{3}}{\sqrt{\sigma^{2}-1}|\underline{q}|^{4 \epsilon} \hbar}\left(\frac{4\left(1+2 \sigma^{2}\right)}{\epsilon^{4}} \mathcal{I}_{2}(\sigma)-\frac{4 \sigma \sqrt{\sigma^{2}-1}}{\epsilon^{3}} \mathcal{I}_{4}(\sigma)+\frac{\left(2 \sigma^{2}-1\right)^{2}}{2 \epsilon^{4}} \mathcal{I}_{6}(\sigma)\right)+\mathcal{O}\left(\epsilon^{0}\right) .
$$

Using the evaluation of the master $\mathcal{I}_{2}(\sigma)$ and $\mathcal{I}_{4}(\sigma)$ in section 5.2 , and $\mathcal{I}_{6}(\sigma)$ in section 5.6 in [32] we obtain

$$
\mathcal{M}_{2}^{H}=\frac{8 \pi G_{N}^{3} m_{1}^{3} m_{2}^{3}\left(4 \pi e^{-\gamma_{E}}\right)^{2 \epsilon}}{\epsilon \sqrt{\sigma^{2}-1}|q|^{4 \epsilon} \hbar}\left(\left(3+12 \sigma^{2}-4 \sigma^{4}\right) \operatorname{arccosh}(\sigma)-4 \sigma \sqrt{\sigma^{2}-1}\right)+\mathcal{O}\left(\hbar^{0}\right) .
$$

Notice that the $H$-diagram integral starts contributing from the classical order in the $\hbar \rightarrow 0$ limit. The full $H$-diagram has recently been considered in ref. [34].

\subsection{Box-bubble contribution}

After tensorial reduction we find for the box-bubble numerator,

$$
\mathcal{M}_{2}^{\square \circ}=-\frac{i 2 G_{N}^{3} m_{1}^{3} m_{2}^{3}\left(2 \sigma^{2}-1\right)\left(1+522 \sigma^{2}\right)\left(4 \pi e^{-\gamma_{E}}\right)^{2 \epsilon}}{15 \epsilon^{2} \sqrt{\sigma^{2}-1}|q|^{4 \epsilon} \hbar}+\mathcal{O}\left(\hbar^{0}\right),
$$

which also contributes from the classical order in the $\hbar \rightarrow 0$ limit.

\section{Self-energy diagrams and vertex corrections}

So far, all parts of the amplitude have followed from the three-graviton cut alone. This includes pieces that belong to both the conservative part and the radiation-reaction part. 


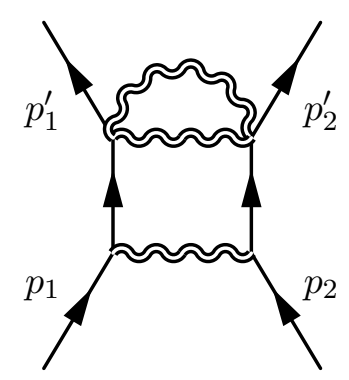

Figure 5. The box-bubble diagram.

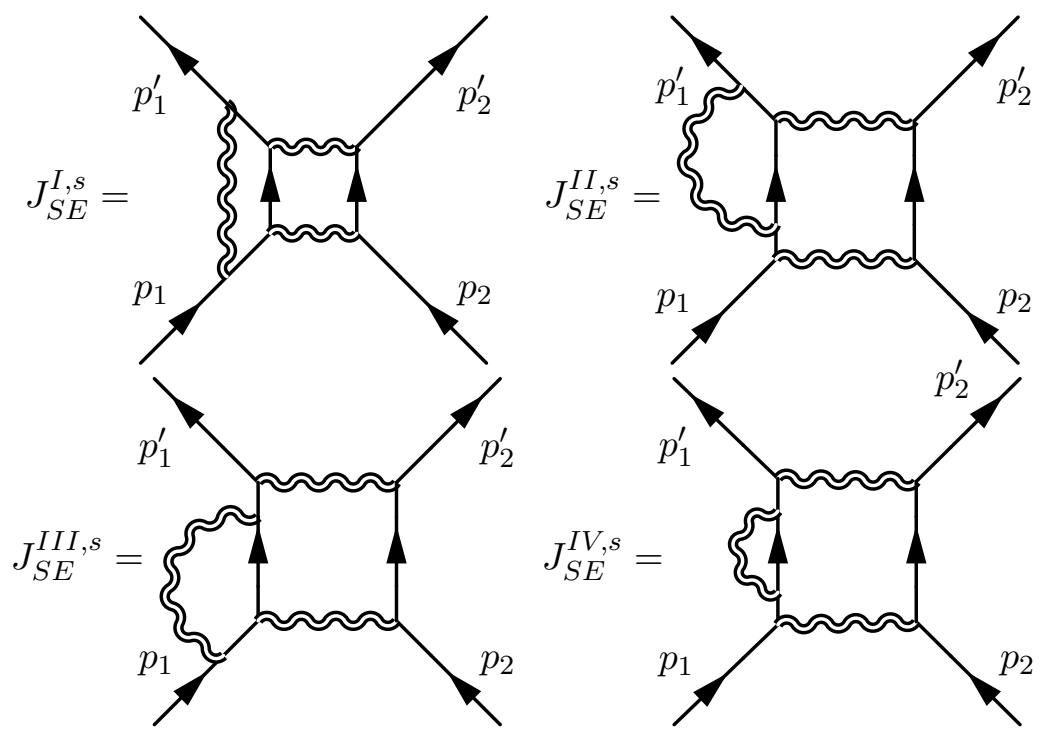

Figure 6. Self-energy diagrams $s$-channel with the graviton line attached the one scalar line.

To get the full set of radiation-reaction contributions, we finally have to compute the two-loop integrals of self-energy and vertex corrections for the massive lines that have not been included in the three-graviton cut. These contributions are not present in the maximal supergravity amplitude because they are subleading in $\hbar \underline{q}$. In that case, the radiation-reaction contribution is solely given by the contribution in (3.14). They each have seven massive propagators (diagrams with fever propagators do not contribute to the radiation-reaction, as they are subleading in $\epsilon$ ). There are four independent sevenpropagator diagrams listed in figure 6 in the $s$-channel with a graviton line attached to the scalar line with mass $m_{1}$. There are four equivalent diagrams with the graviton propagator attached to the other scalar line with mass $m_{2}$. There are as well eight corresponding diagrams in what we can call the $u$-channel (corresponding to the crossed box).

The four associated integrals are

$$
\begin{aligned}
J_{S E}^{I, s}= & 4096 \pi^{3} G_{N}^{3} \hbar^{7} \int \frac{d^{D} l_{1} d^{D} l_{2}}{(2 \pi)^{2 D}} \frac{m_{1}^{8} m_{2}^{4}\left(2 \sigma^{2}-1\right)^{2}+2 m_{1}^{6} m_{2}^{4}\left(2 \sigma^{2}-1\right)^{2}|\hbar q|^{2}}{\left(\left(p_{1}-l_{1}-l_{2}\right)^{2}-m_{1}^{2}+i \varepsilon\right)\left(\left(p_{1}-l_{2}\right)^{2}-m_{1}^{2}+i \varepsilon\right)} \\
& \times \frac{1}{\left(\left(p_{1}-l_{1}-l_{2}-q\right)^{2}-m_{1}^{2}+i \varepsilon\right)\left(\left(p_{2}-l_{1}\right)^{2}-m_{2}^{2}+i \varepsilon\right) l_{1}^{2}\left(l_{1}+q\right)^{2}\left(l_{1}+l_{2}\right)^{2}},
\end{aligned}
$$




$$
\begin{aligned}
J_{S E}^{I I, s}= & 4096 \pi^{3} G_{N}^{3} \hbar^{7} \int \frac{d^{D} l_{1} d^{D} l_{2}}{(2 \pi)^{2 D}} \frac{m_{1}^{8} m_{2}^{4}\left(2 \sigma^{2}-1\right)^{2}}{\left(\left(p_{1}-l_{1}-l_{2}\right)^{2}-m_{1}^{2}+i \varepsilon\right)\left(\left(p_{1}-l_{2}\right)^{2}-m_{1}^{2}+i \varepsilon\right)} \\
& \times \frac{1}{\left(\left(p_{1}+l_{1}\right)^{2}-m_{1}^{2}+i \varepsilon\right)\left(\left(p_{2}-l_{1}\right)^{2}-m_{2}^{2}+i \varepsilon\right) l_{1}^{2}\left(l_{1}+q\right)^{2}\left(l_{1}+l_{2}\right)^{2}}, \\
J_{S E}^{I I I, s}= & 4096 \pi^{3} G_{N}^{3} \hbar^{7} \int \frac{d^{D} l_{1} d^{D} l_{2}}{(2 \pi)^{2 D}} \frac{m_{1}^{8} m_{2}^{4}\left(2 \sigma^{2}-1\right)^{2}}{\left(\left(p_{1}+l_{1}\right)^{2}-m_{1}^{2}+i \varepsilon\right)\left(\left(p_{1}-l_{2}\right)^{2}-m_{1}^{2}+i \varepsilon\right)} \\
& \times \frac{1}{\left(\left(p_{1}-l_{1}-l_{2}-q\right)^{2}-m_{1}^{2}+i \varepsilon\right)\left(\left(p_{2}-l_{1}\right)^{2}-m_{2}^{2}+i \varepsilon\right) l_{1}^{2}\left(l_{1}+q\right)^{2}\left(l_{1}+l_{2}\right)^{2}}, \\
J_{S E}^{I V, s}= & 4096 \pi^{3} G_{N}^{3} \hbar^{7} \int \frac{d^{D} l_{1} d^{D} l_{2}}{(2 \pi)^{2 D}} \frac{m_{1}^{8} m_{2}^{4}\left(2 \sigma^{2}-1\right)^{2}}{\left(\left(p_{1}+l_{1}\right)^{2}-m_{1}^{2}+i \varepsilon\right)^{2}\left(\left(p_{1}-l_{2}\right)^{2}-m_{1}^{2}+i \varepsilon\right)} \\
& \times \frac{1}{\left(\left(p_{2}-l_{1}\right)^{2}-m_{2}^{2}+i \varepsilon\right) l_{1}^{2}\left(l_{1}+q\right)^{2}\left(l_{1}+l_{2}\right)^{2}},
\end{aligned}
$$

with the numerators keeping only contributions that have weight $m_{2}^{4}$ in $p_{2}$ because the other contributions are leading to vanishing contributions having at least two delta-functions on $p_{1} \cdot l$ propagators. As well, only the part of the numerator that is independent of the loop momenta contributes to the classical piece in the $\epsilon$-expansion. Summing all the contributions

$$
\mathcal{M}_{S E}=-2^{16} \pi^{3} G_{N}^{3} \sum_{i=I}^{I V}\left(J_{S E}^{i, s}+J_{S E}^{i, u}\right)+\left(m_{1} \leftrightarrow m_{2}\right),
$$

the self-energy contribution is proportional the master integrals $\mathcal{I}_{5}(\sigma)$ evaluated in section 5 of [32] (see appendix D for a summary of the results)

$$
\begin{aligned}
\mathcal{M}_{S E} & =-\frac{1408 \pi^{3} G_{N}^{3} m_{1}^{3} m_{2}^{3}\left(2 \sigma^{2}-1\right)^{2}}{3 \epsilon^{3} \sqrt{\sigma^{2}-1}|q|^{4 \epsilon} \hbar} \mathcal{I}_{5}(\sigma)+\mathcal{O}\left(\epsilon^{-1}\right) \\
& =i \frac{44 \pi G_{N}^{3} m_{1}^{3} m_{2}^{3}\left(2 \sigma^{2}-1\right)^{2}}{3 \epsilon^{2}\left(\sigma^{2}-1\right)^{\epsilon+\frac{1}{2}}|q|^{4 \epsilon} \hbar}\left(\frac{-1}{4}\right)^{\epsilon}\left(4 \pi e^{-\gamma_{E}}\right)^{2 \epsilon}+\mathcal{O}\left(\epsilon^{-1}\right) .
\end{aligned}
$$

\section{The two-loop amplitude and the eikonal phase}

Summing up the three-particle cut and the self-energy diagrams and vertex corrections, we obtain for the total amplitude

$$
\mathcal{M}_{2}(\sigma,|\underline{q}|)=\frac{1}{|\underline{q}|^{4 \epsilon}}\left(\mathcal{M}_{2}^{(-3)}(\sigma,|\underline{q}|)+\mathcal{M}_{2}^{(-2)}(\sigma,|\underline{q}|)+\mathcal{M}_{2}^{(-1)}(\sigma,|\underline{q}|)+\mathcal{O}\left(\hbar^{0}\right)\right),
$$

with the super-classical pieces

$$
\mathcal{M}_{2}^{(-3)}(\sigma,|\underline{q}|)=-\frac{8 \pi G_{N}^{3} m_{1}^{4} m_{2}^{4}\left(2 \sigma^{2}-1\right)^{3} \Gamma(-\epsilon)^{3} \Gamma(1+2 \epsilon)}{3 \hbar^{3}|\underline{q}|^{2}\left(\sigma^{2}-1\right)(4 \pi)^{-2 \epsilon} \Gamma(-3 \epsilon)},
$$

and

$$
\mathcal{M}_{2}^{(-2)}(\sigma,|\underline{q}|)=\frac{6 i \pi^{2} G_{N}^{3}\left(m_{1}+m_{2}\right) m_{1}^{3} m_{2}^{3}\left(2 \sigma^{2}-1\right)\left(1-5 \sigma^{2}\right)\left(4 \pi e^{-\gamma_{E}}\right)^{2 \epsilon}}{\epsilon \sqrt{\sigma^{2}-1} \hbar^{2}|\underline{q}|}+\mathcal{O}\left(\epsilon^{0}\right),
$$


together with the classical term to the order $1 / \epsilon$ for the real part and $1 / \epsilon^{2}$ for the imaginary terms

$$
\begin{aligned}
\mathcal{M}_{2}^{(-1)}(\sigma, \mid \underline{q})= & \frac{2 \pi G_{N}^{3}\left(4 \pi e^{-\gamma_{E}}\right)^{2 \epsilon} m_{1}^{2} m_{2}^{2}}{\hbar \epsilon}\left(\frac{s\left(2 \sigma^{2}-1\right)^{3}}{\left(\sigma^{2}-1\right)^{2}}\right. \\
& +\frac{i m_{1} m_{2}\left(2 \sigma^{2}-1\right)}{\pi \epsilon\left(\sigma^{2}-1\right)^{\frac{3}{2}}}\left(\frac{1-49 \sigma^{2}+18 \sigma^{4}}{15}-\frac{2 \sigma\left(7-20 \sigma^{2}+12 \sigma^{4}\right) \operatorname{arccosh}(\sigma)}{\sqrt{\sigma^{2}-1}}\right) \\
& -\frac{3\left(2 \sigma^{2}-1\right)\left(1-5 \sigma^{2}\right) s}{2\left(\sigma^{2}-1\right)}+\frac{1}{2}\left(m_{1}^{2}+m_{2}^{2}\right)\left(18 \sigma^{2}-1\right)-\frac{1}{3} m_{1} m_{2} \sigma\left(103+2 \sigma^{2}\right) \\
& +\frac{4 m_{1} m_{2}\left(3+12 \sigma^{2}-4 \sigma^{4}\right) \operatorname{arccosh}(\sigma)}{\sqrt{\sigma^{2}-1}} \\
& \left.-\frac{2 i m_{1} m_{2}\left(2 \sigma^{2}-1\right)^{2}}{\pi \epsilon \sqrt{\sigma^{2}-1}}\left(\frac{-1}{4\left(\sigma^{2}-1\right)}\right)^{\epsilon}\left(-\frac{11}{3}+\frac{d}{d \sigma}\left(\frac{\left(2 \sigma^{2}-1\right) \operatorname{arccosh}(\sigma)}{\sqrt{\sigma^{2}-1}}\right)\right)\right) .
\end{aligned}
$$

The last line gives the radiation-reaction contributions

$$
\begin{aligned}
\left.\mathcal{M}_{2}^{(-1)}(\sigma,|\underline{q}|)\right|_{\text {Rad. }}= & -\frac{4 i G_{N}^{3}\left(4 \pi e^{-\gamma_{E}}\right)^{2 \epsilon} m_{1}^{3} m_{2}^{3}}{\hbar \epsilon^{2}} \frac{\left(2 \sigma^{2}-1\right)^{2}}{\sqrt{\sigma^{2}-1}}\left(\frac{-1}{4\left(\sigma^{2}-1\right)}\right)^{\epsilon} \\
& \times\left(-\frac{11}{3}+\frac{d}{d \sigma}\left(\frac{\left(2 \sigma^{2}-1\right) \operatorname{arccosh}(\sigma)}{\sqrt{\sigma^{2}-1}}\right)\right) .
\end{aligned}
$$

The $-11 / 3$ comes solely from the self-energy diagrams of section 4 and the derivative term from the double-box diagrams of section 3.1. The real and imaginary parts of this term clearly satisfies the relation conjectured in $[16,17]$

$$
\lim _{\epsilon \rightarrow 0} \epsilon \operatorname{Re}(5.5)=-\lim _{\epsilon \rightarrow 0} \epsilon^{2} \pi \operatorname{Im}(5.5) .
$$

\subsection{The amplitude in $b$-space and eikonal exponentiation}

The amplitude is $b$-space is defined by

$$
\widetilde{\mathcal{M}}_{2}(\sigma, b)=\frac{1}{4 E_{\mathrm{c} . \mathrm{m} .} P} \int_{\mathbb{R}^{D-2}} \frac{d^{D-2} \overrightarrow{\underline{q}}}{(2 \pi)^{D-2}} \mathcal{M}_{2}\left(p_{1}, p_{2}, p_{1}^{\prime}, p_{2}^{\prime}\right) e^{i \overrightarrow{\vec{q}} \cdot \vec{b}},
$$

where $4 E_{\text {c.m. }} P=4 m_{1} m_{2} \sqrt{\sigma^{2}-1}$ and $E_{\text {c.m. }}=\sqrt{s}$.

The two-loop amplitude in (5.1) naturally decomposes as follows after Fourier transform to $b$-space

$$
\begin{aligned}
\widetilde{\mathcal{M}}_{2}(\sigma, b)=- & \frac{1}{6}\left(\widetilde{\mathcal{M}}_{0}^{(-1)}(\sigma, b)\right)^{3}+i \widetilde{\mathcal{M}}_{0}^{(-1)}(\sigma, b)\left(\widetilde{\mathcal{M}}_{1}^{\mathrm{Cl}} \cdot(\sigma, b)+\widetilde{\mathcal{M}}_{1}^{\mathrm{Qt} .}(\sigma, b)\right) \\
& +\widetilde{\mathcal{M}}_{2}^{\mathrm{Cl} .}(\sigma, b)+\mathcal{O}\left(\hbar^{0}\right) .
\end{aligned}
$$

We note the following identifications, observed already at the level of diagram topologies:

$$
\widetilde{\mathcal{M}}_{2}^{\square(-3)}(\sigma, b)=-\frac{1}{6}\left(\widetilde{\mathcal{M}}_{0}^{(-1)}(\sigma, b)\right)^{3},
$$




$$
\begin{aligned}
\widetilde{\mathcal{M}}_{2}^{\square(-2)}(\sigma, b)= & i \widetilde{\mathcal{M}}_{0}^{(-1)}(\sigma, b) \widetilde{\mathcal{M}}_{1}^{\square(-1)}(\sigma, b), \\
\widetilde{\mathcal{M}}_{2}^{\triangleleft}(-2)(\sigma, b)+\widetilde{\mathcal{M}}_{2}^{\square \triangleright(-2)}(\sigma, b)= & i \widetilde{\mathcal{M}}_{0}^{(-1)}(\sigma, b)\left(\widetilde{\mathcal{M}}_{1}^{\triangleleft(-1)}(\sigma, b)+\widetilde{\mathcal{M}}_{1}^{\triangleright(-1)}(\sigma, b)\right), \\
\widetilde{\mathcal{M}}_{2}^{\square(-1)}(\sigma, b)= & i \widetilde{\mathcal{M}}_{0}^{(-1)}(\sigma, b) \widetilde{\mathcal{M}}_{1}^{\square(0)}(\sigma, b)+\widetilde{\mathcal{M}}_{2}^{\square \mathrm{Cl}} .(\sigma, b), \\
\widetilde{\mathcal{M}}_{2}^{\square(-1)}(\sigma, b)+\widetilde{\mathcal{M}}_{2}^{\square \triangleright(-1)}(\sigma, b)= & i \widetilde{\mathcal{M}}_{0}^{(-1)}(\sigma, b)\left(\widetilde{\mathcal{M}}_{1}^{\triangleleft(0)}(\sigma, b)+\widetilde{\mathcal{M}}_{1}^{\triangleright(0)}(\sigma, b)\right) \\
& +\widetilde{\mathcal{M}}_{2}^{\triangleleft} \mathrm{Cl} .(\sigma, b)+\widetilde{\mathcal{M}}_{2}^{\square \triangleright \mathrm{Cl} .}(\sigma, b), \\
\widetilde{\mathcal{M}}_{2}^{\square \circ(-1)}(\sigma, b)= & i \widetilde{\mathcal{M}}_{0}^{(-1)}(\sigma, b) \widetilde{\mathcal{M}}_{1}^{\circ(0)}(\sigma, b)+\widetilde{\mathcal{M}}_{2}^{\square \circ} \mathrm{Cl} .(\sigma, b),
\end{aligned}
$$

where

$$
\widetilde{\mathcal{M}}_{0}^{(-1)}(\sigma, b)=\frac{G_{N} m_{1} m_{2}\left(2 \sigma^{2}-1\right) \Gamma(-\epsilon)}{\sqrt{\sigma^{2}-1} \hbar}\left(\pi b^{2}\right)^{\epsilon},
$$

is the first Post-Minkowskian contribution.

The various pieces from the one-loop amplitude $\mathcal{M}_{1}$ are detailed in appendix C. In the above expressions, $\widetilde{\mathcal{M}}_{1}^{\square(0)}(\sigma, b)$ is the Fourier transform of first quantum contribution from the one-loop boxes in (C.8), $\widetilde{\mathcal{M}}_{1}^{\triangleright(-1)}(\sigma, b)$ is the Fourier transform of the classical piece from the one-loop triangle in $(\mathrm{C} .14)$ and $\widetilde{\mathcal{M}}_{1}^{\triangleright(0)}(\sigma, b)$ is the Fourier transform of the first quantum correction from the one-loop triangle in (C.15) (likewise for $\widetilde{\mathcal{M}}_{1}^{\triangleleft(-1)}(\sigma, b)$ and $\left.\widetilde{\mathcal{M}}_{1}^{\triangleleft(0)}(\sigma, b)\right)$, and finally $\widetilde{\mathcal{M}}_{1}^{\circ(0)}(\sigma, b)$ is the Fourier transform of (C.19).

It is striking how the above factorizations arise within graph topologies. This will be explained in section 5.2 below.

Collecting the classical and the leading quantum pieces of the one-loop amplitude as in (C.20), we obtain after Fourier transform to $b$-space the classical piece

$$
\widetilde{\mathcal{M}}_{1}^{\mathrm{Cl}} \cdot(\sigma, b)=\frac{3 \pi G_{N}^{2}\left(m_{1}+m_{2}\right) m_{1} m_{2}\left(5 \sigma^{2}-1\right)}{4 b \sqrt{\sigma^{2}-1} \hbar}\left(\pi b^{2} e^{\gamma_{E}}\right)^{2 \epsilon}+\mathcal{O}(\epsilon)
$$

and the leading quantum correction

$$
\begin{aligned}
\widetilde{\mathcal{M}}_{1}^{\mathrm{Qt} .}(\sigma, b)= & \frac{G_{N}^{2}\left(\pi b^{2} e^{\gamma_{E}}\right)^{2 \epsilon}}{b^{2}}\left(\frac{i \epsilon s\left(2 \sigma^{2}-1\right)^{2}}{\left(\sigma^{2}-1\right)^{2}}\right. \\
& \left.-\frac{m_{1} m_{2}}{\pi\left(\sigma^{2}-1\right)^{\frac{3}{2}}}\left(\frac{1-49 \sigma^{2}+18 \sigma^{4}}{15}-\frac{2 \sigma\left(2 \sigma^{2}-1\right)\left(6 \sigma^{2}-7\right) \operatorname{arccosh}(\sigma)}{\sqrt{\sigma^{2}-1}}\right)\right) .
\end{aligned}
$$

The first and the last term in this expression matches the one derived in [27], the second term arises from the contributions of the triangle and bubble in the Einstein gravity oneloop amplitude as detailed in appendix C. ${ }^{3}$

\footnotetext{
${ }^{3}$ We note that the static limit of the second line matches the quantum correction to the one-loop amplitude evaluated in $[35,36]$$$
\lim _{\sigma \rightarrow 1} \frac{1}{2\left(\sigma^{2}-1\right)}\left(\frac{1-49 \sigma^{2}+18 \sigma^{4}}{15}-\frac{2 \sigma\left(2 \sigma^{2}-1\right)\left(6 \sigma^{2}-7\right) \operatorname{arccosh}(\sigma)}{\sqrt{\sigma^{2}-1}}\right)=-\frac{41}{10} .
$$ 
Finally, we have defined the classical third Post-Minkowskian contribution ${ }^{4}$

$$
\begin{aligned}
\widetilde{\mathcal{M}}_{2}^{\mathrm{Cl} .}(\sigma, b) \equiv & \widetilde{\mathcal{M}}_{2}^{\square \mathrm{Cl} .}(\sigma, b)+\widetilde{\mathcal{M}}_{2}^{\triangleleft} \mathrm{Cl} .(\sigma, b)+\widetilde{\mathcal{M}}_{2}^{\square}{ }^{\mathrm{Cl} .}(\sigma, b) \\
& +\widetilde{\mathcal{M}}_{2}^{\triangleleft \triangleleft} \mathrm{Cl} .+\widetilde{\mathcal{M}}_{2}^{\triangleright \triangleright \mathrm{Cl} .}+\widetilde{\mathcal{M}}_{2}^{H} \mathrm{Cl} .+\widetilde{\mathcal{M}}_{2}^{S E \mathrm{Cl} .}+\widetilde{\mathcal{M}}_{2}^{\square \circ \mathrm{Cl} .}(\sigma, b) \\
= & \frac{G_{N}^{3} m_{1} m_{2}\left(\pi b^{2} e^{\gamma_{E}}\right)^{3 \epsilon}}{\hbar b^{2} \sqrt{\sigma^{2}-1}}\left(\frac{3\left(2 \sigma^{2}-1\right)\left(5 \sigma^{2}-1\right) s}{2\left(\sigma^{2}-1\right)}+\frac{s-2 m_{1} m_{2} \sigma}{2}\left(18 \sigma^{2}-1\right)\right. \\
& -\frac{1}{3} m_{1} m_{2} \sigma\left(103+2 \sigma^{2}\right)+\frac{4 m_{1} m_{2}\left(3+12 \sigma^{2}-4 \sigma^{4}\right) \operatorname{arccosh}(\sigma)}{\sqrt{\sigma^{2}-1}} \\
& \left.-\frac{2 i m_{1} m_{2}\left(2 \sigma^{2}-1\right)^{2}}{\pi \epsilon \sqrt{\sigma^{2}-1}}\left(\frac{-1}{4\left(\sigma^{2}-1\right)}\right)^{\epsilon}\left(-\frac{11}{3}+\frac{d}{d \sigma}\left(\frac{\left(2 \sigma^{2}-1\right) \operatorname{arccosh}(\sigma)}{\sqrt{\sigma^{2}-1}}\right)\right)\right),
\end{aligned}
$$

the last line of this expression is the radiation-reaction part which matches the result of [17].

\subsection{Relation to the world-line formalism: velocity cuts}

As we have remarked earlier, the two-loop amplitude can be organized with integrals involving delta functions as in our earlier case of maximal supergravity [32]. There is a simple correspondence between the order in the $\hbar$ expansion and the number of delta-function insertions. Namely, symbolically we have the following pattern of delta-function insertions

$$
\begin{aligned}
\mathcal{M}_{2}^{(-3)} \sim & \frac{1}{\hbar^{3} \underline{q}^{2}} \int \delta\left(p_{1} \cdot l_{1}\right) \delta\left(p_{1} \cdot l_{2}\right) \delta\left(p_{2} \cdot l_{1}\right) \delta\left(p_{2} \cdot l_{2}\right), \\
\mathcal{M}_{2}^{(-2)} \sim & \frac{1}{\hbar^{2} \underline{q}} \int\left(\delta\left(p_{1} \cdot l_{1}\right) \delta\left(p_{1} \cdot l_{2}\right) \delta\left(p_{2} \cdot l_{1}\right)+\delta\left(p_{1} \cdot l_{1}\right) \delta\left(p_{2} \cdot l_{1}\right) \delta\left(p_{2} \cdot l_{2}\right)\right), \\
\mathcal{M}_{2}^{(-1)} \sim & \frac{1}{\hbar} \int\left(\delta\left(p_{1} \cdot l_{1}\right) \delta\left(p_{2} \cdot l_{1}\right)+\delta\left(p_{1} \cdot l_{1}\right) \delta\left(p_{1} \cdot l_{2}\right)\right) \\
& +\frac{1}{\hbar} \int\left(\delta\left(p_{1} \cdot l_{1}\right) \delta\left(p_{2} \cdot l_{2}\right)+\delta\left(p_{2} \cdot l_{1}\right) \delta\left(p_{2} \cdot l_{2}\right)\right) .
\end{aligned}
$$

We remark that only the inverse powers of $\hbar$ can be localised using delta-functions. The quantum contributions, starting from the order $\hbar^{0}$, are not reducible to $D-1$ dimensional integrals.

Because the delta functions project loop momenta onto external momenta, and hence external four-velocities, we shall call the action of such delta functions velocity cuts. They are not true $D$-dimensional cuts but they share many features with genuine cuts, especially after Fourier transformation into $b$-space. Indeed, these velocity cuts do not decouple the integrals in momentum space but certain parts of them lead to factorized forms in $b$-space. This amplitude factorization is important for the exponentiation of the eikonal phase. As will be explained next, we can readily follow the diagrammatics of this factorization in $b$-space by tracing out how the cuts are distributed. We indicate a velocity cut by a red dashed bar across a massive line.

\footnotetext{
${ }^{4}$ The classical part $\widetilde{\mathcal{M}}_{2}^{\square} \circ \mathrm{Cl} .(\sigma, b)$ vanishes and does contribute to this result. The amplitude $\widetilde{\mathcal{M}}_{2}^{\square \circ(-1)}(\sigma, b)$ is only needed for the eikonalisation of the two-loop amplitude as shown in eq. (5.16).
} 
For instance, two velocity cuts (i.e., two delta-function insertions) in the following diagram
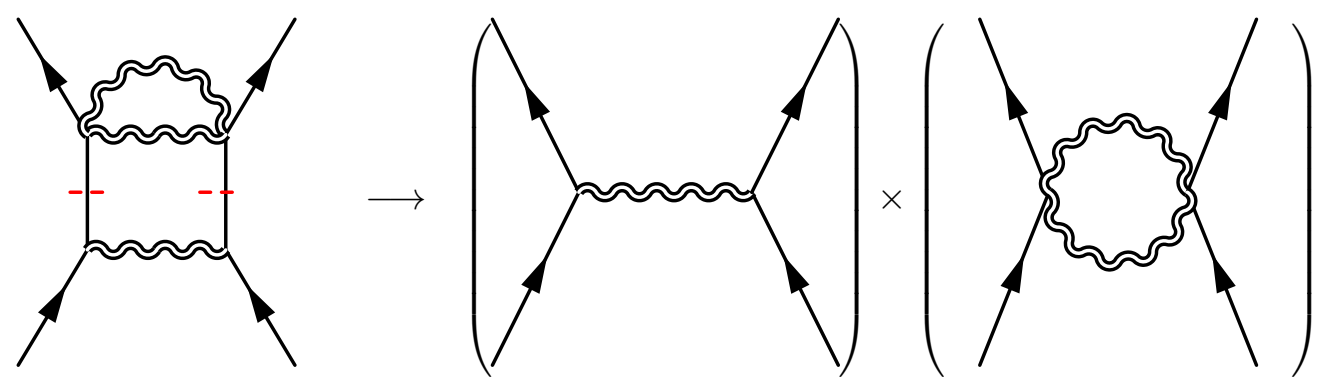

will in $b$-space lead to the product of the tree-level amplitude and a one-loop bubble integral, so that the integral becomes

$$
\begin{aligned}
\widetilde{\mathcal{M}}_{2}^{\square \circ(-1)} & =i\left(-\frac{G_{N} m_{1} m_{2}\left(2 \sigma^{2}-1\right)\left(\pi b^{2}\right)^{\epsilon}}{\hbar \sqrt{\sigma^{2}-1}}\right) \times\left(\frac{G_{N}^{2} m_{1} m_{2}\left(1+522 \sigma^{2}\right)\left(\pi b^{2}\right)^{2 \epsilon}}{15 \pi b^{2} \sqrt{\sigma^{2}-1}}\right) \\
& =i \widetilde{\mathcal{M}}_{0}^{(-1)}(\sigma, b) \times \widetilde{\mathcal{M}}_{1}^{\circ(0)}(\sigma, b),
\end{aligned}
$$

since $\widetilde{\mathcal{M}_{2}}{ }^{\circ}$ Cl. $(\sigma, b)$ vanishes because the two velocity-cuts are taken regarding the same internal momenta.

Two velocity cuts of the box-triangle integral in figure 7 lead, after a Fourier transform to $b$-space, to a product of the tree-level amplitude and a triangle integral in exactly the same way, ${ }^{5}$ plus an extra term contributing to the eikonal phase. As one final example, let us consider two velocity cuts in the double-box integral in figure 8. After a Fourier transformation to $b$-space, this leads to the product of a tree-level amplitude times a oneloop box, in addition to the extra pieces shown.

This graphical interpretation of the action of the delta functions also has another advantage that we wish to explain. In ref. [3] we showed how to relate the second quantized field theory analysis to that of the world-line formalism. In the conservative sector and when integrals are evaluated in the potential region only, this connection arises from extracting the residues of the time-components of the loop momenta. The velocity cuts that we have illustrated in this two-loop calculation can be viewed as a covariant generalization of that reduction in dimensionality of the loop integrals. For an $n$-loop amplitude the most important set of graph topologies in this respect is that of just $n$ massive propagators. When we apply velocity cuts on such graphs all massive propagators will have been acted upon and what is left can be reinterpreted as external sources after a Fourier transform. In figure 9 we draw those two-loop topologies which correspond to just two massive propagators. As each velocity cut removes one loop integration and effectively reduces the matter line to two external sources we denote them by blobs in the same figure. The remaining three-dimensional integrals are what appear as a spatial Fourier transform in the world-line formalism. One of these integrations can be taken to represent the parametrized world line and the remaining two integrations are analogous to the transformation into $b$-space.

\footnotetext{
${ }^{5}$ In particular we see the correspondence between the first line of (3.17) and the quantum correction from the one-loop triangle in (C.15).
} 

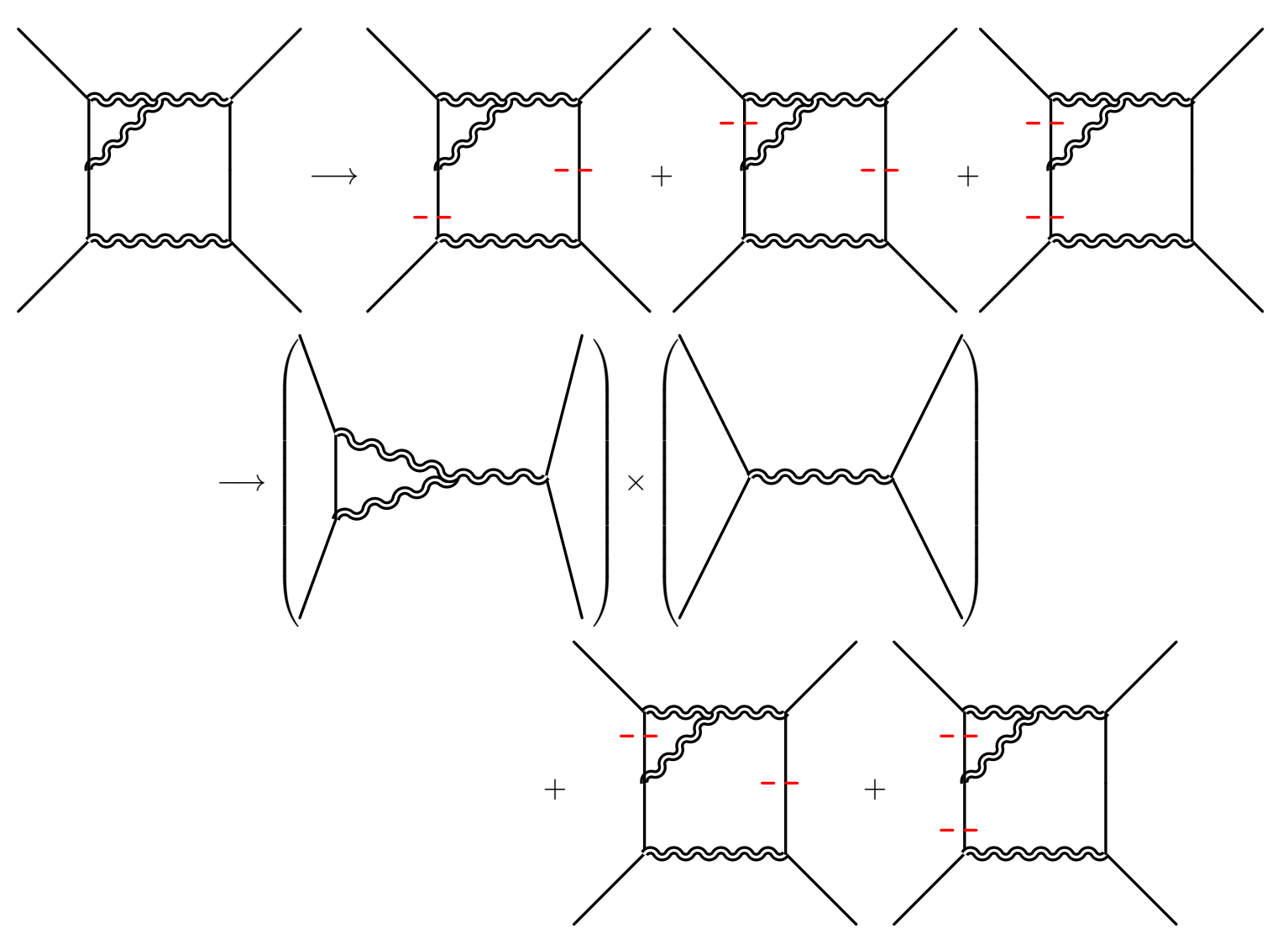

Figure 7. Velocity cuts of the box-triangle diagrams.

Recently, Kälin, Liu, and Porto [10] (see also refs. [37-39]) have shown how to derive the conservative part of the Post-Minkowskian scattering angle to the third Post-Minkowskian order considered here. The diagrams needed are precisely those listed in figure 9 . There are obviously many other diagrams contributing to the (conservative) classical part of the full amplitude but those that factorize do not contribute to physical observables and are eliminated by either the Born subtractions, or the effective field theory matching, or the eikonal exponentiation, depending on which framework one prefers. Indeed, the world-line formalism works at the level of the effective action and hence lives already in the exponent, without any need of subtractions. This still leaves certain terms left over after the factorization and those diagrams (which still have un-cut massive propagators because they stem from topologies with, for $n$-loop graphs, with more than $n$ massive propagators) do not appear to have a simple diagrammatic interpretation in world-line language, although they are of course there. Graph topologies corresponding to radiationreaction contributions also have more than $n$ massive propagators and will therefore also not immediately be amenable to this kind of world-line interpretation. It is interesting to note that the recent calculation of the conservative part at fourth Post-Minkowskian order [8] is organized along similar lines of the exponentiated action and it should therefore match quite directly to the world-line formalism at that order. 


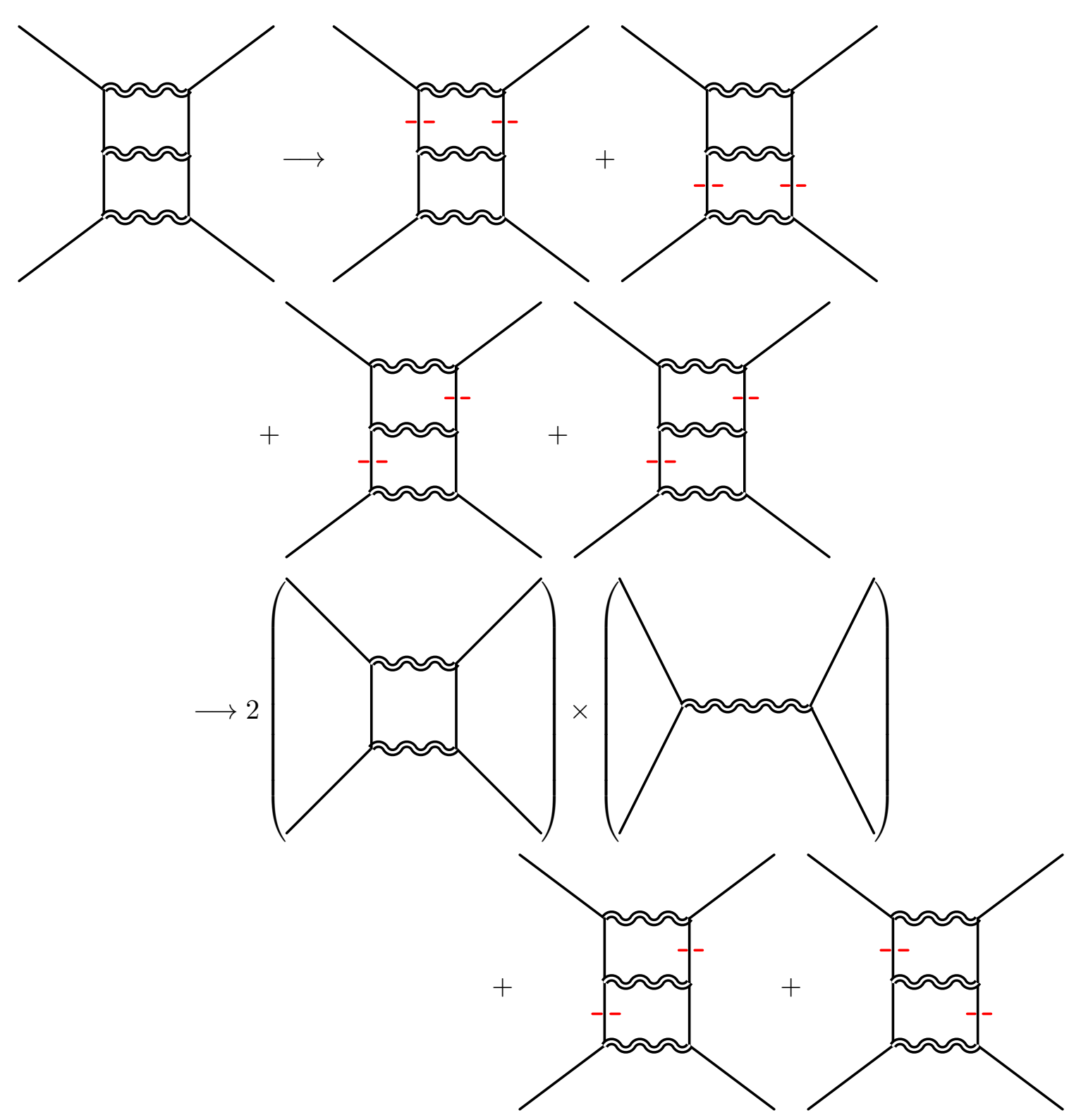

Figure 8. Velocity cuts of the double-box diagram.

The correspondence between our present computation and the calculation at the same order in the world-line formalism appears to run deeper than this. Indeed, the basis of integrals used in ref. [10] coincides with ours, through the following translation table: the set

$$
\left\{I_{11111}, I_{11211}, I_{01101}, I_{11011}, I_{00211}, I_{00112}, I_{00111}\right\}
$$

of eq. (16) in ref. [10] corresponds to

$$
\left\{\mathcal{I}^{0,0,1,1,1,1,1}, \mathcal{I}^{0,0,1,1,1,1,2}, \mathcal{I}^{0,0,0,1,0,1,1}, \mathcal{I}^{0,0,1,1,1,1,0}, \mathcal{I}^{0,0,0,0,1,1,2}, \mathcal{I}^{0,0,0,0,1,2,1}, \mathcal{I}^{0,0,0,0,1,1,1}\right\}
$$

in our notation of equation (5.10) in [32]. This basis of master integrals suffices for the part of the conservative sector that is immediately translatable into those velocity cut diagrams 


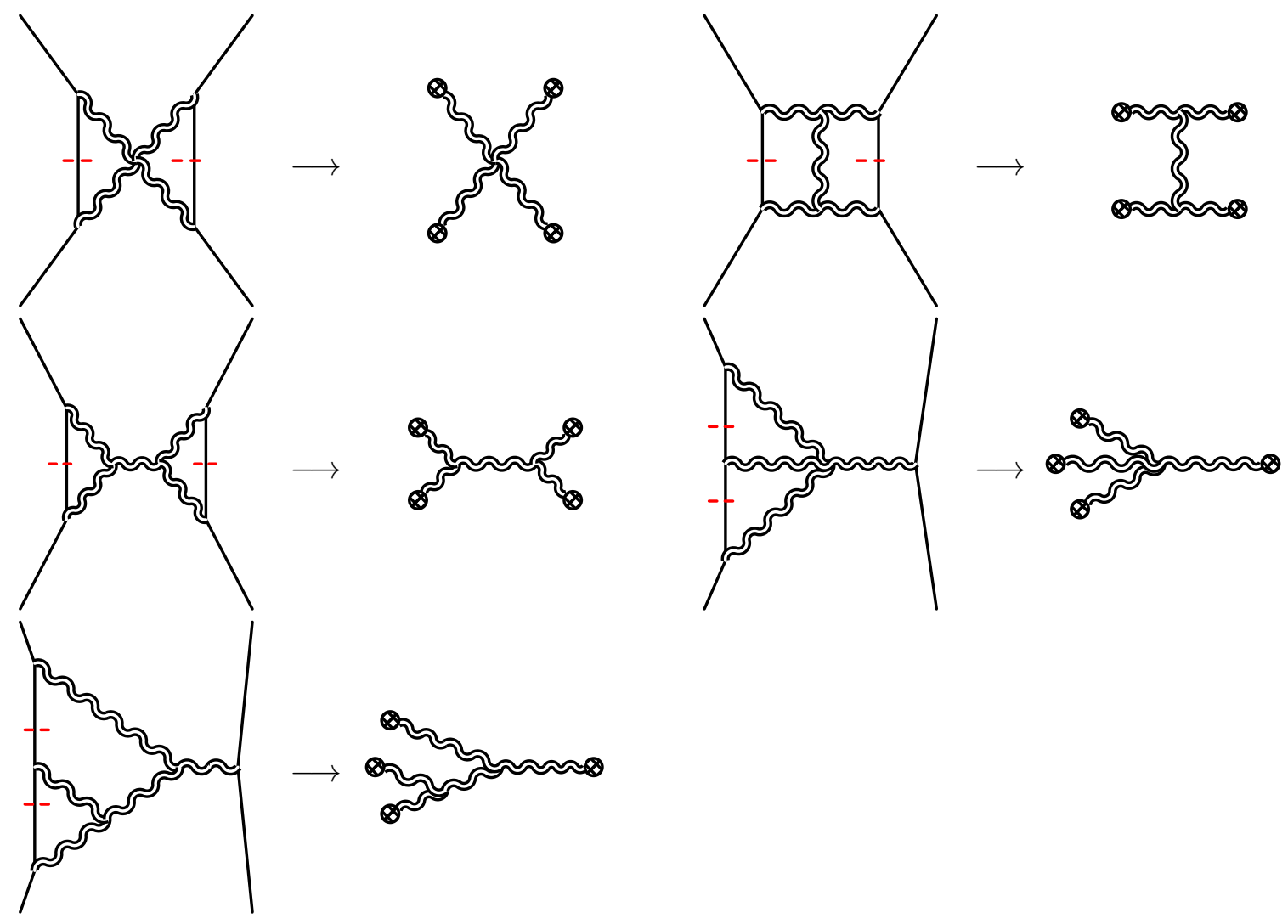

Figure 9. Correspondence with world-line diagrams.

that have matches to standard world-line diagrams. We note that $\mathcal{I}^{0,0,0,1,0,1,1}=0$ is set to zero in the analysis of $[10,21]$, and indeed it only contributes to the radiation-reaction terms. In ref. [10] two more basis integrals were needed. They were denoted by

$$
\left\{M_{11,11100}^{(1,2)}, M_{11,11100}^{(1,1)}\right\},
$$

in eq. (18) of ref. [10] and they correspond to our remaining two

$$
\left\{\mathcal{I}^{1,1,0,0,1,1,1}, \mathcal{I}^{1,1,0,0,1,1,1}(\sigma=1)\right\},
$$

from ref. [32]. This demonstrates explicitly the one-to-one match of the two bases. It is as expected for the diagrams for the conservative sector since we have seen the translation table between graph topologies. But as we have shown here, just the same basis of integrals also encapsulates all radiation-reaction parts. Since the basis of master integrals contains also the crucial terms from what is known as the soft region of the integrals, it does suggest that radiation reaction also has a natural interpretation in world-line language. At higher orders in the Post-Minkowskian expansion we expect the corresponding choice of a minimal basis of master integrals to be quite crucial in order to simplify calculations.

\subsection{The scattering angle}

We can now evaluate the eikonal phase at third Post-Minkowskian order. The classical limit of the two-loop two-body scattering in (5.8) takes the form needed for the exponentation 
of the classical third Post-Minkowskian eikonal in (5.13)

$$
1+i \sum_{L \geq 0} \widetilde{\mathcal{M}}_{L}(\sigma, b)=(1+2 i \Delta(\sigma, b)) \exp \left(\frac{2 i}{\hbar} \sum_{L \geq 0} \delta_{L}(\sigma, b)\right) .
$$

Thanks to the factorized form of the two-loop classical contribution in (5.8) we have the first Post-Minkowskian eikonal phase

$$
\delta_{0}(\sigma, b)=\frac{\hbar}{2}(5.10)=-\frac{G_{N} m_{1} m_{2}\left(2 \sigma^{2}-1\right)}{2 \epsilon \sqrt{\sigma^{2}-1}}\left(\pi b^{2} e^{\gamma_{E}}\right)^{\epsilon}+\mathcal{O}(\epsilon),
$$

the second Post-Minkowskian eikonal phase

$$
\delta_{1}(\sigma, b)=\frac{\hbar}{2}(5.11)=\frac{3 \pi G_{N}^{2}\left(m_{1}+m_{2}\right) m_{1} m_{2}\left(5 \sigma^{2}-1\right)}{8 b \sqrt{\sigma^{2}-1}}\left(\pi b^{2} e^{\gamma_{E}}\right)^{2 \epsilon},
$$

and the third Post-Minkowskian eikonal phase from the real part of the third PostMinkowskian amplitude

$$
\begin{aligned}
\delta_{2}(\sigma, b)= & \frac{\hbar}{2} \operatorname{Re}(5.13)=\frac{G_{N}^{3} m_{1} m_{2}\left(\pi b^{2} e^{\gamma_{E}}\right)^{3 \epsilon}}{2 b^{2} \sqrt{\sigma^{2}-1}}\left(\frac{2 s\left(12 \sigma^{4}-10 \sigma^{2}+1\right)}{\sigma^{2}-1}\right. \\
& -\frac{4 m_{1} m_{2} \sigma}{3}\left(25+14 \sigma^{2}\right)+\frac{4 m_{1} m_{2}\left(3+12 \sigma^{2}-4 \sigma^{4}\right) \operatorname{arccosh}(\sigma)}{\sqrt{\sigma^{2}-1}} \\
& \left.+\frac{2 m_{1} m_{2}\left(2 \sigma^{2}-1\right)^{2}}{\sqrt{\sigma^{2}-1}} \frac{1}{\left(4\left(\sigma^{2}-1\right)\right)^{\epsilon}}\left(-\frac{11}{3}+\frac{d}{d \sigma}\left(\frac{\left(2 \sigma^{2}-1\right) \operatorname{arccosh}(\sigma)}{\sqrt{\sigma^{2}-1}}\right)\right)\right) .
\end{aligned}
$$

And the leading quantum corrections at one-loop

$$
2 \Delta_{1}=\widetilde{\mathcal{M}}_{1}^{\mathrm{Qt}} \cdot(\sigma, b)
$$

The third Post-Minkowskian order scattering angle is then obtained as

$$
\left.\sin \left(\frac{\chi}{2}\right)\right|_{3 P M}=-\frac{\sqrt{s}}{m_{1} m_{2} \sqrt{\sigma^{2}-1}} \frac{\partial \delta_{2}(\sigma, b)}{\partial b},
$$

giving

$$
\begin{aligned}
\left.\sin \left(\frac{\chi}{2}\right)\right|_{3 P M}= & \frac{G_{N}^{3} \sqrt{s}}{b^{3}\left(\sigma^{2}-1\right)}\left(\frac{3\left(2 \sigma^{2}-1\right)\left(5 \sigma^{2}-1\right) s}{2\left(\sigma^{2}-1\right)}\right. \\
& +\frac{m_{1}^{2}+m_{2}^{2}}{2}\left(18 \sigma^{2}-1\right)-\frac{m_{1} m_{2}}{3} \sigma\left(103+2 \sigma^{2}\right)+\frac{4 m_{1} m_{2}\left(3+12 \sigma^{2}-4 \sigma^{4}\right) \operatorname{arccosh}(\sigma)}{\sqrt{\sigma^{2}-1}} \\
& \left.+\frac{2 m_{1} m_{2}\left(2 \sigma^{2}-1\right)^{2}}{\pi \sqrt{\sigma^{2}-1}} \frac{1}{\left(4\left(\sigma^{2}-1\right)\right)}\left(-\frac{11}{3}+\frac{d}{d \sigma}\left(\frac{\left(2 \sigma^{2}-1\right) \operatorname{arccosh}(\sigma)}{\sqrt{\sigma^{2}-1}}\right)\right)\right)+\mathcal{O}(\epsilon)
\end{aligned}
$$

Using the definition of the angular momentum

$$
J=\frac{m_{1} m_{2} \sqrt{\sigma^{2}-1}}{\sqrt{s}} b \cos \left(\frac{\chi}{2}\right)
$$


we obtain for the scattering angle at the first and second Post-Minkowskian order

$$
\begin{aligned}
\chi_{1 P M} & =\frac{2 G_{N} m_{1} m_{2}\left(2 \sigma^{2}-1\right)}{J \sqrt{\sigma^{2}-1}}, \\
\chi_{2 P M} & =\frac{3 \pi G_{N}^{2} m_{1}^{2} m_{2}^{2}\left(m_{1}+m_{2}\right)\left(5 \sigma^{2}-1\right)}{4 J^{2} \sqrt{s}},
\end{aligned}
$$

and at the third Post-Minkowksian order we obtain

$$
\chi_{3 P M}=\widehat{\chi}_{3 P M}+\chi_{3 P M}^{\text {Rad. }},
$$

with

$$
\begin{aligned}
\widehat{\chi}_{3 P M}= & \frac{2 G_{N}^{3} m_{1}^{3} m_{2}^{3}\left(64 \sigma^{6}-120 \sigma^{4}+60 \sigma^{2}-5\right)}{3 J^{3}\left(\sigma^{2}-1\right)^{\frac{3}{2}}} \\
& +\frac{8 G_{N}^{3} m_{1}^{4} m_{2}^{4} \sqrt{\sigma^{2}-1}}{3 J^{3} s}\left(\sigma\left(-25-14 \sigma^{2}\right)+\frac{3\left(3+12 \sigma^{2}-4 \sigma^{4}\right) \operatorname{arccosh}(\sigma)}{\sqrt{\sigma^{2}-1}}\right),
\end{aligned}
$$

from the first and the second line of (5.20), matching the expressions in [2, 5, 40]. And the radiation-reaction part from the third line of $(5.20)$

$$
\chi_{3 P M}^{\mathrm{Rad} .}=\frac{4 G_{N}^{3} m_{1}^{4} m_{2}^{4}\left(2 \sigma^{2}-1\right)^{2}}{J^{3} s} \frac{1}{\left(4\left(\sigma^{2}-1\right)\right)^{\epsilon}}\left(-\frac{11}{3}+\frac{d}{d \sigma}\left(\frac{\left(2 \sigma^{2}-1\right) \operatorname{arccosh}(\sigma)}{\sqrt{\sigma^{2}-1}}\right)\right) .
$$

This expression matches the results of $[15,16,20]$.

\section{Conclusion}

Using the integration method proposed in ref. [32], we have evaluated the two-to-two massive scattering amplitude of two scalars in Einstein gravity, keeping all terms that are needed to compute the classical scattering angle at third Post-Minkowskian order. The expansion is ordered as a Laurent expansion in $\hbar$, but can also be broken into pieces corresponding to the conservative sector and additional pieces that have recently been identified as radiation-reaction contributions. Our results have confirmed all aspects of the recent work in refs. [14-17].

An important simplification of our calculation stems from the fact that the same small basis of master integrals that recently was used to solve the corresponding problem in maximal supergravity [32] can be used in Einstein gravity as well. Similarly, the reduction of dimensionality in the integrals due to a specific grouping of integrands works in Einstein gravity just as it does in maximal supergravity. This gives us the link to the worldline formalism through what we have dubbed velocity cuts. We anticipate that these simplifications apply to higher orders in the Post-Minkowskian expansion as well.

\section{Acknowledgments}

The research of P.V. has received funding from the ANR grant "Amplitudes" ANR-17CE31-0001-01, and the ANR grant "SMAGP" ANR-20-CE40-0026-01 and is partially supported by Laboratory of Mirror Symmetry NRU HSE, RF Government grant, ag. No 
14.641.31.0001. P.V. is grateful to the I.H.E.S. for the use of their computer resources. The work of P.H.D. was supported in part by DFF grant 0135-00089A. The work of N.E.J.B.-B. was supported in part by the Carlsberg Foundation.

We also acknowledge interesting discussions with participants at the GGI workshop "Gravitational scattering, inspiral, and radiation", April-May, 2021.

\section{A The five-point tree amplitude and the three-graviton cut}

To compute the cut integral, we need the five points amplitude where a massive scalar emits three gravitons. The result for these contributions is obtained by computing the tree amplitudes using Feynman diagrams, and was confirmed using the double copy relations (see [41] for a review) from the QCD amplitude of the emission of three gluons from a massive scalar [42]. This amplitude can be put in a simple form using the helicity formalism (see [43] for a review), and subsequently considering the two independent helicity configurations [44].

The singlet amplitude.

$$
i \mathcal{M}_{0}\left(p_{1}, p_{1}^{\prime}, l_{1}^{+}, l_{2}^{+}, l_{3}^{+}\right)=-\frac{\left(8 \pi G_{N}\right)^{\frac{3}{2}} m_{1}^{4}}{\left\langle l_{1} l_{2}\right\rangle^{2}\left\langle l_{1} l_{3}\right\rangle^{2}\left\langle l_{2} l_{3}\right\rangle^{2}} \sum_{1 \leq i \neq j \neq k \leq 3} \frac{\left(l_{i} \cdot l_{j}\right)\left(l_{j} \cdot l_{k}\right) t r_{+}\left[l_{k}, p_{1}, p_{1}^{\prime}, l_{i}\right]}{\left(p_{1} \cdot l_{k}\right)\left(p_{1}^{\prime} \cdot l_{i}\right)}
$$

with $i \mathcal{M}_{0}\left(p_{1}, p_{1}^{\prime}, l_{1}^{-}, l_{2}^{-}, l_{3}^{-}\right)$obtained by complex conjugation. The singlet amplitude is the one given in [45]. This amplitude vanishes when $m_{1}=0$.

We have defined

$$
t r_{ \pm}(a b c d) \equiv 2(a \cdot b c \cdot d-a \cdot c b \cdot d+a \cdot d b \cdot c) \pm 2 i \epsilon^{\mu \nu \rho \sigma} a_{\mu} b_{\nu} c_{\rho} d_{\sigma} .
$$

The non-singlet amplitude.

$$
\begin{aligned}
i \mathcal{M}_{0}\left(p_{1}, p_{1}^{\prime}, l_{1}^{-}, l_{2}^{+}, l_{3}^{+}\right)=\frac{\left(2 \pi G_{N}\right)^{\frac{3}{2}}}{2}( & \sum_{2 \leq j \neq k \leq 3} \frac{\left\langle l_{1}\left|p_{1}\right| l_{j}\right]\left\langle l_{1}\left|p_{1}^{\prime}\right| l_{j}\right]^{2}\left\langle l_{1}\left|p_{1}\right| l_{k}\right]^{3}}{\left\langle l_{1} l_{j}\right\rangle\left\langle l_{1} l_{k}\right\rangle\left(l_{1} \cdot l_{j}\right)\left(l_{1} \cdot l_{k}\right)\left(p_{1} \cdot l_{1}\right)\left(p_{1}^{\prime} \cdot l_{j}\right)} \\
& -\frac{\left\langle l_{1}\left|p_{1}\right| l_{2}\right]^{3}\left\langle l_{1}\left|p_{1}^{\prime}\right| l_{3}\right]^{3}}{\left\langle l_{1} l_{2}\right\rangle\left\langle l_{1} l_{3}\right\rangle\left(l_{1} \cdot l_{2}\right)\left(l_{1} \cdot l_{3}\right)\left(p_{1} \cdot l_{2}\right)\left(p_{1}^{\prime} \cdot l_{3}\right)} \\
& -\frac{2\left[l_{2} l_{3}\right]\left\langle l_{1}\left|p_{1}\right| l_{2}\right]\left\langle l_{1}\left|p_{1}\right| l_{3}\right]\left\langle l_{1}\left|p_{1}\right| p_{1}^{\prime} \mid l_{1}\right\rangle^{2}}{\left\langle l_{1} l_{2}\right\rangle\left\langle l_{1} l_{3}\right\rangle\left\langle l_{2} l_{3}\right\rangle\left(l_{1} \cdot l_{2}\right)\left(l_{1} \cdot l_{3}\right)\left(p_{1} \cdot l_{1}\right)} \\
& \left.+\frac{2\left[l_{2} l_{3}\right]^{3}\left\langle l_{1}\left|p_{1}\right| p_{1}^{\prime} \mid l_{1}\right\rangle^{2}}{\left\langle l_{2} l_{3}\right\rangle\left(l_{1} \cdot l_{2}\right)\left(l_{1} \cdot l_{3}\right) t}\right)+\left(p_{1} \leftrightarrow-p_{1}^{\prime}\right),
\end{aligned}
$$

with $i \mathcal{M}_{0}\left(p_{1}, p_{1}^{\prime}, l_{1}^{+}, l_{2}^{-}, l_{3}^{-}\right)$obtained by complex conjugation. The advantage of these expressions is that they keep track of the symmetry regarding $p_{1}$ and $-p_{1}^{\prime}$ exchange, and regarding the internal momenta $l_{1}, l_{2}$ and $l_{3}$ satisfying $l_{1}+l_{2}+l_{3}+q=0$.

The three-particle cut. The three-particle cut in (2.7) is obtained by summing the singlet and the non-singlet contributions

$$
\mathcal{M}_{2}^{3-\text { cut }}\left(\sigma, q^{2}\right)=\left.\mathcal{M}_{2}^{3-\text { cut }}\left(\sigma, q^{2}\right)\right|_{\text {singlet }}+\left.\mathcal{M}_{2}^{3-\text { cut }}\left(\sigma, q^{2}\right)\right|_{\text {non-singlet }},
$$


where

$$
\begin{aligned}
\left.\mathcal{M}_{2}^{3-\text { cut }}\left(\sigma, q^{2}\right)\right|_{\text {singlet }}= & \int \frac{d^{D} l_{1} d^{D} l_{2}}{(2 \pi)^{2 D}} \delta\left(l_{1}+l_{2}+l_{3}+q\right) \frac{i^{3}}{l_{1}^{2} l_{2}^{2} l_{3}^{2}} \\
& \times \frac{1}{3 !}\left(\sum_{\operatorname{Perm}\left(l_{1}, l_{2}, l_{3}\right)} \mathcal{M}_{0}\left(p_{1}, p_{1}^{\prime}, l_{1}^{+}, l_{1}^{+}, l_{3}^{+}\right) \mathcal{M}_{0}\left(p_{2}, p_{2}^{\prime}, l_{1}^{-}, l_{1}^{-}, l_{3}^{-}\right)\right. \\
& \left.+\frac{1}{3 !} \sum_{\operatorname{Perm}\left(l_{1}, l_{2}, l_{3}\right)} \mathcal{M}_{0}\left(p_{1}, p_{1}^{\prime}, l_{1}^{-}, l_{1}^{-}, l_{3}^{-}\right) \mathcal{M}_{0}\left(p_{2}, p_{2}^{\prime}, l_{1}^{+}, l_{1}^{+}, l_{3}^{+}\right)\right),
\end{aligned}
$$

and the non-singlet cut

$$
\begin{aligned}
\left.\mathcal{M}_{2}^{3-\text { cut }}\left(\sigma, q^{2}\right)\right|_{\text {non-singlet }}= & \int \frac{d^{D} l_{1} d^{D} l_{2}}{(2 \pi)^{2 D}} \delta\left(l_{1}+l_{2}+l_{3}+q\right) \frac{i^{3}}{l_{1}^{2} l_{2}^{2} l_{3}^{2}} \\
& \times\left(\frac{1}{3 !} \sum_{\operatorname{Perm}\left(l_{1}, l_{2}, l_{3}\right)} \mathcal{M}_{0}\left(p_{1}, p_{1}^{\prime}, l_{1}^{-}, l_{1}^{+}, l_{3}^{+}\right) \mathcal{M}_{0}\left(p_{2}, p_{2}^{\prime}, l_{1}^{+}, l_{1}^{-}, l_{3}^{-}\right)\right. \\
& \left.+\frac{1}{3 !} \sum_{\operatorname{Perm}\left(l_{1}, l_{2}, l_{3}\right)} \mathcal{M}_{0}\left(p_{1}, p_{1}^{\prime}, l_{1}^{+}, l_{1}^{-}, l_{3}^{-}\right) \mathcal{M}_{0}\left(p_{2}, p_{2}^{\prime}, l_{1}^{-}, l_{1}^{+}, l_{3}^{+}\right)\right) .
\end{aligned}
$$

\section{B Numerator factors}

In this appendix we list the numerator factors entering the expression of the three-particle cut of section 3. With the individual integral defined by the graphs we obtain the following numerators from the tensorial reduction of the three-particle cut:

The double-box numerators have to be expanded up to order $(\hbar \underline{\vec{q}})^{2}$.

The numerator factor for the double-box integral in the left of figure 1 in the $s$-channel

$$
\mathcal{N}_{\square}^{(s)}=512 \pi^{3} G_{N}^{3}\left(m_{1}^{4}+m_{2}^{4}-2\left(m_{1}^{2}+m_{2}^{2}\right) s+s^{2}\right)^{3}=2^{12} \pi^{3} G_{N}^{3} m_{1}^{6} m_{2}^{6}\left(2 \sigma^{2}-1\right)^{3},
$$

the numerator factor for the crossed double-box integral in the right of figure 1 in the $s$-channel

$$
\mathcal{N}_{\square}^{(\text {cross }, s)}=2^{13} \pi^{3} G_{N}^{3}\left(96 m_{1}^{6} m_{2}^{6}\left(2 \sigma^{2}-1\right)^{3}+8 m_{1}^{5} m_{2}^{5} \sigma\left(2 \sigma^{2}-1\right)^{2}(\hbar \underline{\vec{q}})^{2}\left(l_{2} \cdot l_{3}\right)+\mathcal{O}\left((\hbar \underline{\vec{q}})^{4}\right)\right) .
$$

The numerator for the double-box integral in the $u$-channel is given by

$$
\begin{aligned}
\mathcal{N}_{\square}^{(u)} & =512 \pi^{3} G_{N}^{3}\left(m_{1}^{4}+m_{2}^{4}-2\left(m_{1}^{2}+m_{2}^{2}\right) u+u^{2}\right)^{3} \\
& =2^{12} \pi^{3} G_{N}^{3}\left(96 m_{1}^{6} m_{2}^{6}\left(2 \sigma^{2}-1\right)^{3}-6 m_{1}^{5} m_{2}^{5} \sigma\left(2 \sigma^{2}-1\right)^{2}(\hbar \underline{\vec{q}})^{2}+\mathcal{O}\left((\hbar \underline{\vec{q}})^{4}\right)\right),
\end{aligned}
$$

and for the cross double-box integral in the $u$-channel

$\mathcal{N}_{\square}^{(\text {cross }, u)}=2^{13} \pi^{3} G_{N}^{3}\left(m_{1}^{6} m_{2}^{6}\left(2 \sigma^{2}-1\right)^{3}-8 m_{1}^{5} m_{2}^{5} \sigma\left(2 \sigma^{2}-1\right)^{2}(\hbar \underline{\vec{q}})^{2}\left(l_{2} \cdot l_{3}+\frac{3}{4}\right)+\mathcal{O}\left((\hbar \underline{\vec{q}})^{4}\right)\right)$. 
The box-triangle numerators have to be expanded to the order $|\hbar \underline{q}|$.

The numerator factor for the non-planar box-triangle integral in the right of figure 2 in the $s$-channel is $\mathcal{N}_{\square}^{(N P)}=\mathcal{N}_{\square}^{(N P, I)}+\mathcal{N}_{\square}^{(N P, I I)}$ with

$$
\begin{aligned}
\mathcal{N}_{\square}^{(N P, I)}=1024 \pi^{3} G_{N}^{3} & \left(\frac{m_{1}^{6} m_{2}^{2}\left(2 \sigma^{2}-1\right)\left(\left(p_{2} \cdot l_{1}-p_{2} \cdot l_{2}\right)^{2}+4 m_{2}^{2}\left(1-4 \sigma^{2}\right)\left(l_{1} \cdot l_{2}\right)\right)}{l_{1} \cdot l_{2}}\right. \\
& -\frac{2 m_{1}^{5} m_{2} \sigma(\hbar \underline{\vec{q}})\left(p_{2} \cdot l_{1}-p_{2} \cdot l_{2}\right)\left(2 m_{2}^{2}\left(1-2 \sigma^{2}\right)-\left(p_{2} \cdot l_{1}-p_{2} \cdot l_{2}\right)^{2}+8 m_{2}^{2}\left(3 \sigma^{2}-1\right)\left(l_{1} \cdot l_{2}\right)\right)}{l_{1} \cdot l_{2}} \\
& \left.+\mathcal{O}\left((\hbar \underline{\vec{q}})^{2}\right)\right),
\end{aligned}
$$

and

$$
\begin{aligned}
\mathcal{N}_{\square}^{(N P, I I)}=1024 \pi^{3} G_{N}^{3} & \left(\frac{m_{1}^{6} m_{2}^{2}\left(2 \sigma^{2}-1\right)\left(\left(p_{4} \cdot l_{1}-p_{4} \cdot l_{2}\right)^{2}+4 m_{2}^{2}\left(1-4 \sigma^{2}\right)\left(l_{1} \cdot l_{2}\right)\right)}{l_{1} \cdot l_{2}}\right. \\
& -\frac{2 m_{1}^{5} m_{2} \sigma(\hbar \underline{\vec{q}})\left(p_{2} \cdot l_{1}-p_{2} \cdot l_{2}\right)\left(2 m_{2}^{2}\left(1-2 \sigma^{2}\right)-\left(p_{2} \cdot l_{1}-p_{2} \cdot l_{2}\right)^{2}+8 m_{2}^{2}\left(3 \sigma^{2}-1\right)\left(l_{1} \cdot l_{2}\right)\right)}{l_{1} \cdot l_{2}} \\
& \left.+\mathcal{O}\left((\hbar \underline{\vec{q}})^{2}\right)\right) .
\end{aligned}
$$

The numerator factor for the planar box-triangle integral in the left of figure 2 in the $s$-channel is $\mathcal{N}_{\square}^{(s)}=\mathcal{N}_{\square}^{(s, I)}+\mathcal{N}_{\square}^{(s, I I)}$ with

$$
\begin{aligned}
\mathcal{N}_{\square \triangleright}^{(s, I)}=1024 \pi^{3} G_{N}^{3} & \left(\frac{24 m_{1}^{6} m_{2}^{2}\left(2 \sigma^{2}-1\right)\left(\left(p_{2} \cdot l_{1}-p_{2} \cdot l_{3}\right)^{2}+4 m_{2}^{2}\left(1-4 \sigma^{2}\right)\left(l_{1} \cdot l_{3}\right)\right)}{l_{1} \cdot l_{3}}\right. \\
& \left.-16 m_{1}^{5} m_{2}^{3} \sigma(\hbar \underline{\vec{q}})\left(p_{2} \cdot l_{1}-p_{2} \cdot l_{3}\right)\left(2 \sigma^{2}-1\right)+\mathcal{O}\left((\hbar \underline{\vec{q}})^{2}\right)\right),
\end{aligned}
$$

and

$$
\begin{aligned}
\mathcal{N}_{\square \triangleright}^{(s, I I)}=1024 \pi^{3} G_{N}^{3}( & \frac{m_{1}^{6} m_{2}^{2}\left(2 \sigma^{2}-1\right)\left(\left(p_{4} \cdot l_{1}-p_{4} \cdot l_{3}\right)^{2}+4 m_{2}^{2}\left(1-4 \sigma^{2}\right)\left(l_{1} \cdot l_{3}\right)\right)}{l_{1} \cdot l_{3}} \\
& \left.+16 m_{1}^{5} m_{2}^{3} \sigma(\hbar \underline{\vec{q}})\left(p_{2} \cdot l_{1}-p_{2} \cdot l_{3}\right)\left(2 \sigma^{2}-1\right)+\mathcal{O}\left((\hbar \underline{\vec{q}})^{2}\right)\right) .
\end{aligned}
$$

The numerator factor for the planar box-triangle integral in the left of figure 2 in the $u$-channel is $\mathcal{N}_{\square}^{(u)}=\mathcal{N}_{\square \triangleright}^{(u, I)}+\mathcal{N}_{\square}^{(U, I I)}$ with

$$
\begin{aligned}
\mathcal{N}_{\square}^{(u, I)}=1024 \pi^{3} G_{N}^{3}( & \frac{m_{1}^{6} m_{2}^{2}\left(2 \sigma^{2}-1\right)\left(\left(p_{4} \cdot l_{1}-p_{4} \cdot l_{3}\right)^{2}+4 m_{2}^{2}\left(1-4 \sigma^{2}\right)\left(l_{1} \cdot l_{3}\right)\right)}{l_{1} \cdot l_{3}} \\
& \left.-16 m_{1}^{5} m_{2}^{3} \sigma(\hbar \underline{\vec{q}})\left(p_{2} \cdot l_{1}-p_{2} \cdot l_{3}\right)\left(2 \sigma^{2}-1\right)+\mathcal{O}\left((\hbar \underline{\vec{q}})^{2}\right)\right),
\end{aligned}
$$


and

$$
\begin{aligned}
\mathcal{N}_{\square \triangleright}^{(u, I I)}=1024 \pi^{3} G_{N}^{3}( & \frac{m_{1}^{6} m_{2}^{2}\left(2 \sigma^{2}-1\right)\left(\left(p_{2} \cdot l_{1}-p_{2} \cdot l_{3}\right)^{2}+4 m_{2}^{2}\left(1-4 \sigma^{2}\right)\left(l_{1} \cdot l_{3}\right)\right)}{l_{1} \cdot l_{3}} \\
& \left.+16 m_{1}^{5} m_{2}^{3} \sigma(\hbar \underline{\vec{q}})\left(p_{2} \cdot l_{1}-p_{2} \cdot l_{3}\right)\left(2 \sigma^{2}-1\right)+\mathcal{O}\left((\hbar \underline{\vec{q}})^{2}\right)\right),
\end{aligned}
$$

with similar expression for the numerators of the amplitude $\mathcal{M}_{2}^{\triangleleft}(\sigma, b)$.

The double-triangle numerator. The numerator factor for the double-triangle diagram in figure 3 is given by

$$
\begin{aligned}
\mathcal{N}_{\triangleright}=-1024 \pi^{3} G_{N}^{3}\left(\frac{m_{1}^{6} m_{2}^{2}\left(2 \sigma^{2}-1\right)\left(u_{q} \cdot l_{2}-u_{q} \cdot l_{3}\right)^{2}}{l_{2} \cdot l_{3}}+\frac{m_{1}^{6} m_{2}^{2}\left(2 \sigma^{2}-1\right)\left(u_{q} \cdot l_{1}-u_{q} \cdot l_{3}\right)^{2}}{l_{1} \cdot l_{3}}\right. \\
\left.+\frac{m_{1}^{6} m_{2}^{2}\left(2 \sigma^{2}-1\right)\left(u_{q} \cdot l_{1}-u_{q} \cdot l_{2}\right)^{2}}{\left(l_{1} \cdot l_{2}\right)}-m_{1}^{6} m_{2}^{2}\left(-5+34 \sigma^{2}\right)+\mathcal{O}((\hbar \underline{\vec{q}}))\right) \cdot \quad(\text { B. } 11)
\end{aligned}
$$

The $\boldsymbol{H}$ diagram numerator. The numerator factor for the $H$ diagram in figure 4 is given by

$$
\begin{aligned}
\mathcal{N}_{H}= & \frac{128 \pi^{3} G_{N}^{3}}{3}\left(-48\left(-4 m_{1}^{2} m_{2}^{4}\left(\left(l_{2}+l_{3}\right)^{2}-\left(l_{1}+l_{3}\right)^{2}+4 \sigma^{2}\right)\left(\bar{p}_{1} \cdot l_{2}\right)^{2}\right.\right. \\
& -8 m_{2}^{4}\left(\bar{p}_{1} \cdot l_{2}\right)^{4}+16 m_{1}^{3} m_{2}^{3} \sigma\left(\bar{p}_{1} \cdot l_{2}\right)\left(\bar{p}_{2} \cdot l_{1}\right) \\
& +m_{1}^{4}\left(m _ { 2 } ^ { 4 } \left(-1-2\left(l_{2}+l_{3}\right)^{2}\left(1+\left(l_{2}+l_{3}\right)^{2}\right)-2\left(l_{1}+l_{3}\right)^{2}\left(1+\left(l_{1}+l_{3}\right)^{2}\right)\right.\right. \\
& +4 \sigma^{2}+4\left(\left(l_{2}+l_{3}\right)^{2}+\left(l_{2}+l_{3}\right)^{4}+\left(l_{1}+l_{3}\right)^{2}-2\left(l_{2}+l_{3}\right)^{2}\left(l_{1}+l_{3}\right)^{2}+\left(l_{1}+l_{3}\right)^{4}\right) \sigma^{2} \\
& \left.\left.\left.\left.-4 \sigma^{4}\right)+4 m_{2}^{2}\left(\left(l_{2}+l_{3}\right)^{2}-\left(l_{1}+l_{3}\right)^{2}-4 \sigma^{2}\right)\left(\bar{p}_{2} \cdot l_{1}\right)^{2}-8\left(\bar{p}_{2} \cdot l_{1}\right)^{4}\right)\right)(\hbar \underline{\vec{q}})^{4}+\mathcal{O}\left((\hbar \underline{\vec{q}})^{5}\right)\right) .
\end{aligned}
$$

The box-bubble diagram numerator. The numerator factor for the box diagram in figure 5 is given by

$$
\mathcal{N}_{\square}=\frac{2048 \pi^{3} G_{N}^{3} m_{1}^{4} m_{2}^{4}\left(2 \sigma^{2}-1\right)\left(1+522 \sigma^{2}\right)}{15} .
$$

\section{The one-loop two-body amplitude}

The one-loop two-body scattering is done using the two-particle cuts following the computation in [36]

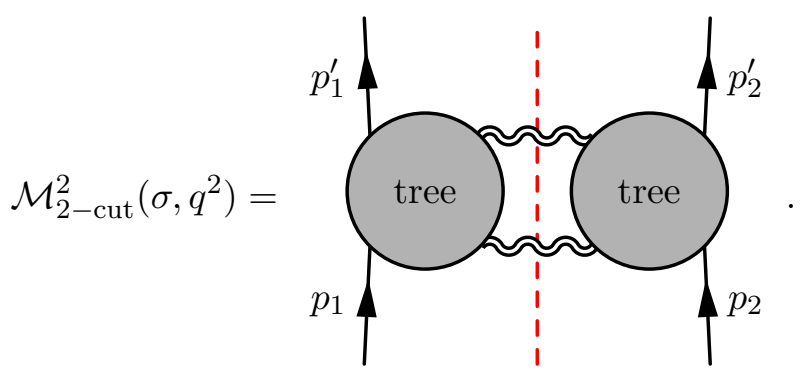


We reduce the tensorial integrals using LiteRed [33], we find that the amplitude decomposes as

$$
\mathcal{M}_{1}\left(\sigma, q^{2}\right)=\mathcal{M}_{1}^{\square}+\mathcal{M}_{1}^{\triangleright}+\mathcal{M}_{1}^{\triangleleft}+\mathcal{M}_{1}^{\circ}
$$

The box contribution is given by

$$
\mathcal{M}_{1}^{\square}\left(\sigma, q^{2}\right)=256 \pi^{2} m_{1}^{4} m_{2}^{4} G_{N}^{2}\left(2 \sigma^{2}-1\right)^{2}\left(I_{s}+I_{u}\right)-1024 \pi^{2} m_{1}^{3} m_{2}^{3} G_{N}^{2}\left(2 \sigma^{2}-1\right)|\hbar \underline{q}|^{2} I_{u},
$$

with the scalar one-loop box and cross-box integrals
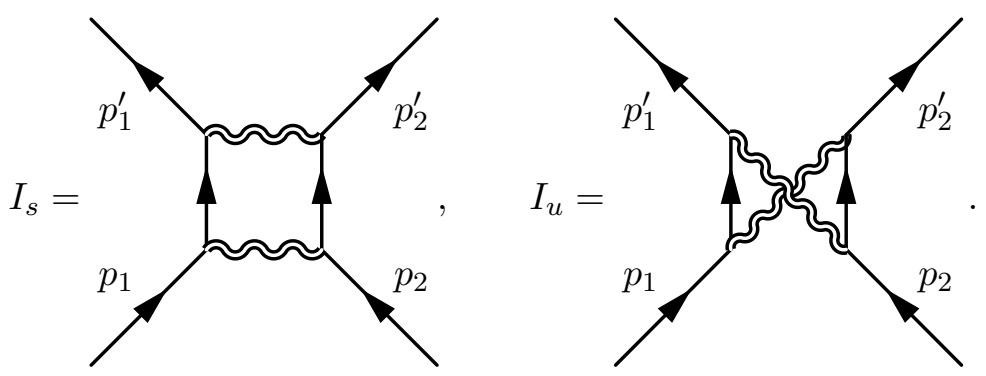

This box part has the following Laurent expansion in $\hbar$ up to the first quantum correction

$$
\mathcal{M}_{1}^{\square}\left(\sigma, q^{2}\right)=\frac{1}{\mid \underline{q}^{2 \epsilon}}\left(\mathcal{M}_{1}^{\square(-2)}+\mathcal{M}_{1}^{\square(-1)}+\mathcal{M}_{1}^{\square(0)}+\mathcal{O}(\hbar)\right),
$$

with

$$
\begin{aligned}
\mathcal{M}_{1}^{\square(-2)}= & -\frac{8 \pi m_{1}^{3} m_{2}^{3} G_{N}^{2}\left(2 \sigma^{2}-1\right)^{2}(4 \pi)^{\epsilon} \Gamma(1+\epsilon) \Gamma(-\epsilon)^{2}}{|\hbar \underline{q}|^{2} \Gamma(-2 \epsilon) \sqrt{\sigma^{2}-1}}, \\
\mathcal{M}_{1}^{\square(-1)}= & 8 \pi^{2} m_{1}^{2} m_{2}^{2} G_{N}^{2}\left(2 \sigma^{2}-1\right)^{2} \frac{i(4 \pi)^{\epsilon}\left(m_{1}+m_{2}\right)}{|\hbar \underline{q}|\left(\sigma^{2}-1\right)} \frac{\Gamma\left(\frac{1}{2}-\epsilon\right)^{2} \Gamma\left(\frac{1}{2}+\epsilon\right)}{\pi^{\frac{3}{2}} \Gamma(-2 \epsilon)}, \\
\mathcal{M}_{1}^{\square(0)}= & 4 m_{1}^{2} m_{2}^{2} G_{N}^{2}\left(2 \sigma^{2}-1\right)^{2} \frac{(4 \pi)^{\epsilon} \Gamma(1+\epsilon) \Gamma(-\epsilon)^{2}}{\left(\sigma^{2}-1\right)^{\frac{3}{2}} \Gamma(-2 \epsilon)} \\
& \times\left(\frac{\epsilon s \pi}{2 m_{1} m_{2}}+i(1+2 \epsilon)\left(\sigma \operatorname{arccosh}(\sigma)-\sqrt{\left.\sigma^{2}-1\right)}\right)\right. \\
& -32 m_{1}^{2} m_{2}^{2} G_{N}^{2}\left(2 \sigma^{2}-1\right) \frac{i(4 \pi)^{\epsilon} \operatorname{arccosh}(\sigma)}{\sqrt{\sigma^{2}-1}} \frac{\Gamma(-\epsilon)^{2} \Gamma(1+\epsilon)}{\Gamma(-2 \epsilon)}|\hbar \underline{q}|^{2} .
\end{aligned}
$$

At the leading order in $\epsilon$ the first quantum correction from the box integral reads

$$
\begin{aligned}
\mathcal{M}_{1}^{\square(0)}= & -\frac{8 G_{N}^{2} m_{1}^{2} m_{2}^{2}\left(2 \sigma^{2}-1\right)\left(4 \pi e^{-\gamma_{E}}\right)^{\epsilon}}{\epsilon|\vec{q}|^{2 \epsilon}\left(\sigma^{2}-1\right)^{\frac{3}{2}}} \\
& \times\left(\frac{\left(2 \sigma^{2}-1\right) \epsilon s \pi}{2 m_{1} m_{2}}+i\left(\left(7-6 \sigma^{2}\right) \operatorname{arccosh}(\sigma)-\left(2 \sigma^{2}-1\right) \sqrt{\sigma^{2}-1}\right)\right) .
\end{aligned}
$$


The triangle contribution. The right-triangle graph

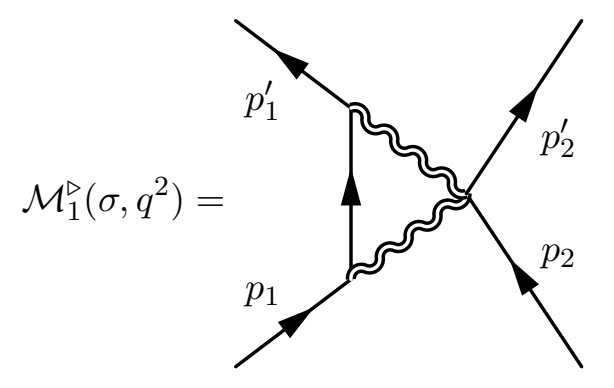

has the following numerator factor

$\mathcal{N}_{\triangleright}=64 \pi^{2} m_{1}^{4} G_{N}^{2}\left(4 m_{2}^{2}\left(4 \sigma^{2}-1\right)+\frac{8\left(p_{2} \cdot l_{1}\right)^{2}}{|\hbar \underline{q}|^{2}}\right)-256 \pi^{2} m_{1}^{3} G^{2}\left(m_{1}+8 m_{2} \sigma\right) p_{2} \cdot l_{1}+\mathcal{O}\left(|\hbar \underline{q}|^{2}\right)$,

which after tensorial reduction leads to

$$
\begin{aligned}
\mathcal{M}_{1}^{\triangleright}\left(\sigma, q^{2}\right)= & 24 i \pi^{2} G_{N}^{2} m_{1}^{4} m_{2}^{2}\left(5 \sigma^{2}-1\right) \int \frac{d^{D} l_{1}}{(2 \pi)^{D-1}} \frac{\delta\left(p_{1} \cdot l_{1}\right)}{l_{1}^{2}\left(l_{1}+q\right)^{2}} \\
& -64 \pi^{2} G_{N}^{2} m_{1}^{2} m_{2}^{2}\left(22 \sigma^{2}-1\right) \int \frac{d^{D} l_{1}}{(2 \pi)^{D}} \frac{1}{l_{1}^{2}\left(l_{1}+q\right)^{2}} .
\end{aligned}
$$

The Laurent expansion in $\hbar$ up to the first quantum correction reads

$$
\mathcal{M}_{1}^{\triangleright}\left(\sigma, q^{2}\right)=\frac{1}{|q|^{2 \epsilon}}\left(\mathcal{M}_{1}^{\triangleright(-1)}+\mathcal{M}_{1}^{\triangleright(0)}+\mathcal{O}(\hbar)\right),
$$

with the classical and first quantum contributions

$$
\begin{aligned}
\mathcal{M}_{1}^{\triangleright(-1)} & =\frac{3 i \pi^{2} G_{N}^{2} m_{1}^{3} m_{2}^{2}\left(5 \sigma^{2}-1\right)\left(4 \pi e^{-\gamma_{E}}\right)^{\epsilon}}{|\hbar \underline{q}|}, \\
\mathcal{M}_{1}^{\triangleright(0)} & =-\frac{4 i G_{N}^{2} m_{1}^{2} m_{2}^{2}\left(22 \sigma^{2}-1\right) i\left(4 \pi e^{-\gamma_{E}}\right)^{\epsilon}}{\epsilon},
\end{aligned}
$$

with an equivalent expression for the left-triangle graph $\mathcal{M}_{1}^{\triangleleft}\left(\sigma, q^{2}\right)$ with exchanging $m_{1}$ and $m_{2}$.

The bubble contribution.

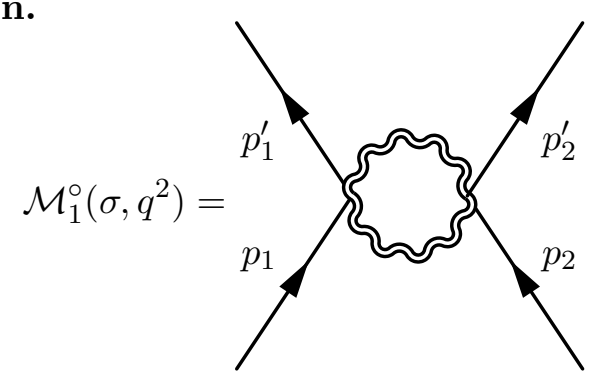

has the numerator factor

$$
\begin{aligned}
\mathcal{N}_{\circ}= & 64 \pi^{2} G_{N}^{2}\left(-4 m_{1}^{2} m_{2}^{2}+40 m_{1}^{2} m_{2}^{2} \sigma^{2}+24 m_{2}^{2}\left(p_{1} \cdot l_{1}\right)^{2}\right. \\
& \left.-64 m_{1} m_{2} \sigma p_{1} \cdot l_{1} p_{2} \cdot l_{1}+24 m_{1}^{2}\left(p_{2} \cdot l_{1}\right)^{2}+16\left(p_{1} \cdot l_{1}\right)^{2}\left(p_{2} \cdot l_{1}\right)^{2}\right)+\mathcal{O}(|\hbar \underline{q}|),
\end{aligned}
$$


which after tensorial reduction leads to

$$
\mathcal{M}_{1}^{\circ}\left(\sigma, q^{2}\right)=\frac{64 \pi^{2} G_{N}^{2} m_{1}^{2} m_{2}^{2}\left(1+522 \sigma^{2}\right)}{15} \int \frac{d^{D} l_{1}}{(2 \pi)^{D}} \frac{1}{l_{1}^{2}\left(l_{1}+q\right)^{2}} .
$$

This starts contributing from the first quantum correction

$$
\mathcal{M}_{1}^{\circ(0)}\left(\sigma, q^{2}\right)=\frac{4 i\left(4 \pi e^{-\gamma_{E}}\right)^{\epsilon} G_{N}^{2} m_{1}^{2} m_{2}^{2}\left(1+522 \sigma^{2}\right)}{15 \epsilon|\underline{q}|^{2 \epsilon}} .
$$

The one-loop amplitude in (C.2), and reads including the first quantum correction

$$
\mathcal{M}_{1}\left(\sigma, q^{2}\right)=\frac{1}{|q|^{2 \epsilon}}\left(\mathcal{M}_{1}^{(-2)}+\mathcal{M}_{1}^{(-1)}+\mathcal{M}_{1}^{(0)}+\mathcal{O}(\hbar)\right)
$$

with

$$
\begin{aligned}
\mathcal{M}_{1}^{(-2)} & =\mathcal{M}_{1}^{\square(-2)}, \\
\mathcal{M}_{1}^{(-1)} & =\mathcal{M}_{1}^{\square(-1)}+\mathcal{M}_{1}^{\triangleright(-1)}+\mathcal{M}_{1}^{\triangleleft(-1)}, \\
\mathcal{M}_{1}^{(0)} & =\mathcal{M}_{1}^{\square(0)}+\mathcal{M}_{1}^{\triangleright(0)}+\mathcal{M}_{1}^{\triangleleft(0)}+\mathcal{M}_{1}^{\circ(0)} .
\end{aligned}
$$

\section{Master integrals}

In this appendix we recall the expressions for the master integrals computed in [32].

With the following definition for the master integral

$$
\begin{aligned}
\mathcal{I}^{n_{1}, n_{2}, n_{3}, n_{4}, n_{5}, n_{6}, n_{7}} \equiv & \int \frac{d^{D-1} l_{1} d^{D-1} l_{2}}{(2 \pi)^{2 D-2}} \frac{1}{\left(k \cdot l_{1}\right)^{n_{1}}\left(k \cdot l_{2}\right)^{n_{2}}\left(l_{1}^{2}\right)^{n_{3}}\left(\left(u_{q}+l_{2}\right)^{2}\right)^{n_{4}}\left(\left(l_{1}-u_{q}\right)^{2}\right)^{n_{5}}} \\
& \times \frac{1}{\left(l_{2}^{2}\right)^{n_{6}}\left(\left(l_{1}+l_{2}\right)^{2}-2(\sigma-1) k \cdot l_{1} k \cdot l_{2}\right)^{n_{7}}},
\end{aligned}
$$

where we have defined $k^{2} \equiv u_{q}^{2} \equiv-1$ and $k \cdot u_{q} \equiv 0$, we collect the results obtained in section 5 of [32].

$$
\mathcal{I}_{1}(\sigma) \equiv 2 \epsilon^{4} \mathcal{I}^{0,0,1,1,1,1,0}=\epsilon^{4} \frac{\Gamma\left(\frac{1}{2}+\epsilon\right)^{2} \Gamma\left(\frac{1}{2}-\epsilon\right)^{4}}{2(4 \pi)^{3-2 \epsilon} \Gamma(1-2 \epsilon)^{2}}
$$

and

$$
\begin{aligned}
\mathcal{I}_{2}(\sigma) \equiv 2 \epsilon^{4} \sqrt{\sigma^{2}-1} \mathcal{I}^{0,0,0,0,1,1,1} \\
=\left(-\frac{2 \epsilon \Gamma\left(\frac{1}{2}-\epsilon\right)^{3} \Gamma(2 \epsilon)}{(4 \pi)^{D-1} \Gamma\left(\frac{3}{2}-3 \epsilon\right)}\left(\sigma^{2}-1\right)^{3 \epsilon} \int_{1}^{\sigma} \frac{d t}{\left(t^{2}-1\right)^{\frac{1}{2}+3 \epsilon}}\right. \\
\left.\quad+2 b_{5}\left(\sigma^{2}-1\right)^{3 \epsilon} \int_{1}^{\sigma} \frac{d t_{2}}{\left(t_{2}^{2}-1\right)^{\frac{1}{2}+3 \epsilon}} \int_{1}^{t_{2}} \frac{d t_{1}}{\left(t_{1}^{2}-1\right)^{\frac{1}{2}+\epsilon}}\right) \epsilon^{3}+\mathcal{O}\left(\epsilon^{4}\right),
\end{aligned}
$$


and

$$
\begin{aligned}
\mathcal{I}_{3}(\sigma)=2 \epsilon^{3} \sqrt{\sigma^{2}-1} \mathcal{I}^{0,0,0,0,1,1,2} & \\
=\frac{-b_{5} \epsilon}{\left(\sigma^{2}-1\right)^{\epsilon}}-( & -4 \frac{b_{5}}{\left(\sigma^{2}-1\right)^{\epsilon}} \int_{1}^{\sigma} \frac{d t_{2}}{\left(t_{2}^{2}-1\right)^{\frac{1}{2}-\epsilon}} \int_{1}^{t_{2}} \frac{d t_{1}}{\left(t_{1}^{2}-1\right)^{\frac{1}{2}+\epsilon}} \\
& \left.\quad+\frac{4 \epsilon \Gamma\left(\frac{1}{2}-\epsilon\right)^{3} \Gamma(2 \epsilon)}{(4 \pi)^{D-1} \Gamma\left(\frac{3}{2}-3 \epsilon\right)} \frac{1}{\left(\sigma^{2}-1\right)^{\epsilon}} \int_{1}^{\sigma} \frac{d t}{\left(t^{2}-1\right)^{\frac{1}{2}-\epsilon}}\right) \epsilon^{3}+\mathcal{O}\left(\epsilon^{4}\right),
\end{aligned}
$$

and

$$
\begin{aligned}
\mathcal{I}_{4}(\sigma) & \equiv 4 \epsilon^{2}\left(\sigma^{2}-1\right) \mathcal{I}^{-1,-1,0,0,1,1,3}+\epsilon^{2}(1+2 \epsilon) \sigma \mathcal{I}^{0,0,0,0,1,1,2} \\
& =\left(-\frac{2 \epsilon \Gamma\left(\frac{1}{2}-\epsilon\right)^{3} \Gamma(2 \epsilon)}{(4 \pi)^{D-1} \Gamma\left(\frac{3}{2}-3 \epsilon\right)}+2 b_{5} \int_{1}^{\sigma} \frac{d t}{\left(t^{2}-1\right)^{\frac{1}{2}+\epsilon}}\right) \epsilon^{2}+\mathcal{O}\left(\epsilon^{4}\right),
\end{aligned}
$$

and

$$
\begin{aligned}
\mathcal{I}_{5}(\sigma) & \equiv \frac{2 \epsilon^{2}(4 \epsilon-1)(2 \epsilon-1)}{\sqrt{\sigma^{2}-1}} \mathcal{I}^{0,0,0,1,0,1,1} \\
& =-\frac{i \epsilon(4 \pi)^{2 \epsilon}}{32 \pi^{2}\left(\sigma^{2}-1\right)^{\epsilon}} \frac{(-1)^{\epsilon} \Gamma(1-2 \epsilon)^{2} \Gamma(1+2 \epsilon)^{2} \Gamma(1-\epsilon)}{\Gamma(1-4 \epsilon) \Gamma(1+\epsilon)},
\end{aligned}
$$

with $(-1)^{\epsilon}=e^{i \pi \epsilon}=1+i \pi \epsilon+\mathcal{O}\left(\epsilon^{2}\right)$, and

$$
\begin{aligned}
\mathcal{I}_{6}(\sigma) & \equiv 2 \epsilon^{4} \sqrt{\sigma^{2}-1} \mathcal{I}^{0,0,1,1,1,1,1} \\
& =\frac{\left(4 \pi e^{-\gamma_{E}}\right)^{2 \epsilon} \epsilon^{3}}{8 \pi^{3}} \operatorname{arcsinh}\left(\sqrt{\frac{\sigma-1}{2}}\right)\left(\pi+2 i\left(\frac{-1}{4\left(\sigma^{2}-1\right)}\right)^{\epsilon} \operatorname{arcsinh}\left(\sqrt{\frac{\sigma-1}{2}}\right)\right)+\mathcal{O}\left(\epsilon^{4}\right),
\end{aligned}
$$

and

$$
\begin{aligned}
\mathcal{I}_{7}(\sigma) & \equiv 8 \epsilon^{4}\left(\sigma^{2}-1\right) \mathcal{I}^{-1,-1,1,1,1,1,1}+4 \epsilon^{4} \sigma \mathcal{I}^{0,0,1,1,1,1,0}, \\
& =0+\mathcal{O}\left(\epsilon^{3}\right),
\end{aligned}
$$

and

$$
\mathcal{I}_{8}(\sigma) \equiv-\epsilon^{3} \mathcal{I}^{0,1,0,0,1,1,2}= \pm \frac{i \sqrt{\pi} \epsilon^{3} \Gamma(-\epsilon) \Gamma\left(-\frac{1}{2}-2 \epsilon\right) \Gamma\left(\frac{3}{2}+2 \epsilon\right) \Gamma\left(\frac{1}{2}-\epsilon\right) \Gamma\left(-\frac{1}{2}-\epsilon\right)}{(4 \pi)^{3-2 \epsilon} \Gamma(-2 \epsilon) \Gamma\left(-\frac{1}{2}-3 \epsilon\right)},
$$

with the + sign for the $+i \varepsilon$ prescription and - for the $+i \varepsilon$ of the linear denominator. We refer to section 5.5.4 of [32] for details. And, finally,

$$
\begin{aligned}
& \mathcal{I}_{9}(\sigma) \equiv \epsilon^{4} \mathcal{I}^{1,1,0,0,1,1,1} \\
&=b_{9} \epsilon^{2}-\frac{b_{5} \epsilon^{2}}{\left(\sigma^{2}-1\right) \epsilon}\left(\operatorname{arccosh}(\sigma)-\epsilon\left(\operatorname{arccosh}(\sigma)^{2}\right.\right. \\
&\left.\left.+\operatorname{Li}_{2}\left(2-2 \sigma\left(\sigma+\sqrt{\sigma^{2}-1}\right)\right)\right)+\mathcal{O}(\epsilon)\right),
\end{aligned}
$$


with

$$
b_{5}=-\frac{i(4 \pi)^{2 \epsilon}}{32 \pi^{2}} \frac{(-1)^{\epsilon} \Gamma(1-2 \epsilon)^{2} \Gamma(1+2 \epsilon)^{2} \Gamma(1-\epsilon)}{\Gamma(1-4 \epsilon) \Gamma(1+\epsilon)},
$$

and $b_{9}$ depends on the $i \varepsilon$ prescription of the linear denominators, with the result (we refer to section 5.5.5 of [32] for details)

$$
b_{9}^{++}=b_{9}^{--}=-2 b_{9}^{+-},
$$

with

$$
b_{9}^{+-}=b_{9}^{-+}=-\frac{\epsilon^{2}}{6} \frac{\Gamma(-\epsilon)^{3} \Gamma(1+2 \epsilon)}{(4 \pi)^{2-2 \epsilon} \Gamma(-3 \epsilon)}=-\frac{\left(4 \pi e^{-\gamma_{E}}\right)^{2 \epsilon}}{32 \pi^{2}}+\mathcal{O}\left(\epsilon^{2}\right) .
$$

Open Access. This article is distributed under the terms of the Creative Commons Attribution License (CC-BY 4.0), which permits any use, distribution and reproduction in any medium, provided the original author(s) and source are credited.

\section{References}

[1] T. Damour, Gravitational scattering, post-Minkowskian approximation and Effective One-Body theory, Phys. Rev. D 94 (2016) 104015 [arXiv: 1609. 00354] [InSPIRE].

[2] T. Damour, High-energy gravitational scattering and the general relativistic two-body problem, Phys. Rev. D 97 (2018) 044038 [arXiv: 1710.10599] [INSPIRE].

[3] N.E.J. Bjerrum-Bohr, P.H. Damgaard, G. Festuccia, L. Planté and P. Vanhove, General Relativity from Scattering Amplitudes, Phys. Rev. Lett. 121 (2018) 171601 [arXiv: 1806.04920] [INSPIRE].

[4] C. Cheung, I.Z. Rothstein and M.P. Solon, From Scattering Amplitudes to Classical Potentials in the Post-Minkowskian Expansion, Phys. Rev. Lett. 121 (2018) 251101 [arXiv: 1808.02489] [INSPIRE].

[5] Z. Bern, C. Cheung, R. Roiban, C.-H. Shen, M.P. Solon and M. Zeng, Scattering Amplitudes and the Conservative Hamiltonian for Binary Systems at Third Post-Minkowskian Order, Phys. Rev. Lett. 122 (2019) 201603 [arXiv: 1901.04424] [INSPIRE].

[6] Z. Bern, C. Cheung, R. Roiban, C.-H. Shen, M.P. Solon and M. Zeng, Black Hole Binary Dynamics from the Double Copy and Effective Theory, JHEP 10 (2019) 206 [arXiv: 1908.01493] [INSPIRE].

[7] C. Cheung and M.P. Solon, Classical gravitational scattering at $\mathcal{O}\left(G^{3}\right)$ from Feynman diagrams, JHEP 06 (2020) 144 [arXiv:2003.08351] [INSPIRE].

[8] Z. Bern et al., Scattering Amplitudes and Conservative Binary Dynamics at $\mathcal{O}\left(G^{4}\right)$, Phys. Rev. Lett. 126 (2021) 171601 [arXiv:2101.07254] [inSPIRE].

[9] A. Cristofoli, N.E.J. Bjerrum-Bohr, P.H. Damgaard and P. Vanhove, Post-Minkowskian Hamiltonians in general relativity, Phys. Rev. D 100 (2019) 084040 [arXiv:1906.01579] [INSPIRE].

[10] G. Kälin, Z. Liu and R.A. Porto, Conservative Dynamics of Binary Systems to Third Post-Minkowskian Order from the Effective Field Theory Approach, Phys. Rev. Lett. 125 (2020) 261103 [arXiv:2007. 04977] [INSPIRE]. 
[11] G. Kälin and R.A. Porto, From Boundary Data to Bound States, JHEP 01 (2020) 072 [arXiv: 1910.03008] [INSPIRE].

[12] N.E.J. Bjerrum-Bohr, A. Cristofoli and P.H. Damgaard, Post-Minkowskian Scattering Angle in Einstein Gravity, JHEP 08 (2020) 038 [arXiv:1910.09366] [INSPIRE].

[13] G. Kälin and R.A. Porto, From boundary data to bound states. Part II. Scattering angle to dynamical invariants (with twist), JHEP 02 (2020) 120 [arXiv: 1911.09130] [INSPIRE].

[14] P. Di Vecchia, C. Heissenberg, R. Russo and G. Veneziano, Universality of ultra-relativistic gravitational scattering, Phys. Lett. B 811 (2020) 135924 [arXiv: 2008.12743] [InSPIRE].

[15] T. Damour, Radiative contribution to classical gravitational scattering at the third order in G, Phys. Rev. D 102 (2020) 124008 [arXiv:2010.01641] [inSPIRE].

[16] P. Di Vecchia, C. Heissenberg, R. Russo and G. Veneziano, Radiation Reaction from Soft Theorems, Phys. Lett. B 818 (2021) 136379 [arXiv:2101.05772] [InSPIRE].

[17] P. Di Vecchia, C. Heissenberg, R. Russo and G. Veneziano, The eikonal approach to gravitational scattering and radiation at $\mathcal{O}\left(G^{3}\right)$, JHEP 07 (2021) 169 [arXiv:2104.03256] [INSPIRE].

[18] D.A. Kosower, B. Maybee and D. O'Connell, Amplitudes, Observables, and Classical Scattering, JHEP 02 (2019) 137 [arXiv: 1811.10950] [INSPIRE].

[19] E. Herrmann, J. Parra-Martínez, M.S. Ruf and M. Zeng, Gravitational Bremsstrahlung from Reverse Unitarity, Phys. Rev. Lett. 126 (2021) 201602 [arXiv:2101.07255] [InSPIRE].

[20] E. Herrmann, J. Parra-Martínez, M.S. Ruf and M. Zeng, Radiative Classical Gravitational Observables at $\mathcal{O}\left(G^{3}\right)$ from Scattering Amplitudes, arXiv:2104.03957 [INSPIRE].

[21] J. Parra-Martínez, M.S. Ruf and M. Zeng, Extremal black hole scattering at $\mathcal{O}\left(G^{3}\right)$ : graviton dominance, eikonal exponentiation, and differential equations, JHEP 11 (2020) 023 [arXiv: 2005.04236] [INSPIRE].

[22] D.N. Kabat and M. Ortiz, Eikonal quantum gravity and Planckian scattering, Nucl. Phys. B 388 (1992) 570 [hep-th/9203082] [INSPIRE].

[23] R. Akhoury, R. Saotome and G. Sterman, High Energy Scattering in Perturbative Quantum Gravity at Next to Leading Power, Phys. Rev. D 103 (2021) 064036 [arXiv:1308.5204] [INSPIRE].

[24] D. Amati, M. Ciafaloni and G. Veneziano, Higher Order Gravitational Deflection and Soft Bremsstrahlung in Planckian Energy Superstring Collisions, Nucl. Phys. B 347 (1990) 550 [INSPIRE].

[25] A. Koemans Collado, P. Di Vecchia, R. Russo and S. Thomas, The subleading eikonal in supergravity theories, JHEP 10 (2018) 038 [arXiv: 1807.04588] [INSPIRE].

[26] M. Ciafaloni, D. Colferai and G. Veneziano, Infrared features of gravitational scattering and radiation in the eikonal approach, Phys. Rev. D 99 (2019) 066008 [arXiv:1812.08137] [INSPIRE].

[27] A. Koemans Collado, P. Di Vecchia and R. Russo, Revisiting the second post-Minkowskian eikonal and the dynamics of binary black holes, Phys. Rev. D 100 (2019) 066028 [arXiv: 1904.02667] [INSPIRE].

[28] P. Di Vecchia, A. Luna, S.G. Naculich, R. Russo, G. Veneziano and C.D. White, A tale of two exponentiations in $\mathcal{N}=8$ supergravity, Phys. Lett. B 798 (2019) 134927 [arXiv: 1908.05603] [INSPIRE]. 
[29] P. Di Vecchia, S.G. Naculich, R. Russo, G. Veneziano and C.D. White, A tale of two exponentiations in $\mathcal{N}=8$ supergravity at subleading level, JHEP 03 (2020) 173 [arXiv: 1911.11716] [INSPIRE].

[30] Z. Bern, H. Ita, J. Parra-Martínez and M.S. Ruf, Universality in the classical limit of massless gravitational scattering, Phys. Rev. Lett. 125 (2020) 031601 [arXiv:2002.02459] [INSPIRE].

[31] A. Cristofoli, P.H. Damgaard, P. Di Vecchia and C. Heissenberg, Second-order Post-Minkowskian scattering in arbitrary dimensions, JHEP 07 (2020) 122 [arXiv: 2003.10274] [INSPIRE].

[32] N.E.J. Bjerrum-Bohr, P.H. Damgaard, L. Planté and P. Vanhove, Classical gravity from loop amplitudes, Phys. Rev. D 104 (2021) 026009 [arXiv:2104.04510] [InSPIRE].

[33] R.N. Lee, LiteRed 1.4: a powerful tool for reduction of multiloop integrals, J. Phys. Conf. Ser. 523 (2014) 012059 [arXiv: 1310.1145] [INSPIRE].

[34] P.A. Kreer and S. Weinzierl, The H-graph with equal masses in terms of multiple polylogarithms, Phys. Lett. B 819 (2021) 136405 [arXiv:2104.07488] [InSPIRE].

[35] N.E.J. Bjerrum-Bohr, J.F. Donoghue and B.R. Holstein, Quantum gravitational corrections to the nonrelativistic scattering potential of two masses, Phys. Rev. D 67 (2003) 084033 [Erratum ibid. 71 (2005) 069903] [hep-th/0211072] [INSPIRE].

[36] N.E.J. Bjerrum-Bohr, J.F. Donoghue and P. Vanhove, On-shell Techniques and Universal Results in Quantum Gravity, JHEP 02 (2014) 111 [arXiv:1309.0804] [INSPIRE].

[37] G. Kälin and R.A. Porto, Post-Minkowskian Effective Field Theory for Conservative Binary Dynamics, JHEP 11 (2020) 106 [arXiv:2006.01184] [INSPIRE].

[38] G. Mogull, J. Plefka and J. Steinhoff, Classical black hole scattering from a worldline quantum field theory, JHEP 02 (2021) 048 [arXiv:2010.02865] [INSPIRE].

[39] G.U. Jakobsen, G. Mogull, J. Plefka and J. Steinhoff, Classical Gravitational Bremsstrahlung from a Worldline Quantum Field Theory, Phys. Rev. Lett. 126 (2021) 201103 [arXiv:2101.12688] [INSPIRE].

[40] A. Antonelli, A. Buonanno, J. Steinhoff, M. van de Meent and J. Vines, Energetics of two-body Hamiltonians in post-Minkowskian gravity, Phys. Rev. D 99 (2019) 104004 [arXiv: 1901.07102] [INSPIRE].

[41] Z. Bern, J.J. Carrasco, M. Chiodaroli, H. Johansson and R. Roiban, The Duality Between Color and Kinematics and its Applications, arXiv:1909.01358 [INSPIRE].

[42] D. Forde and D.A. Kosower, All-multiplicity amplitudes with massive scalars, Phys. Rev. D 73 (2006) 065007 [hep-th/0507292] [INSPIRE].

[43] M.L. Mangano and S.J. Parke, Multiparton amplitudes in gauge theories, Phys. Rept. 200 (1991) 301 [hep-th/0509223] [INSPIRE].

[44] L. Planté, Some aspects on effective field theory of gravity, Ph.D. Thesis, Université Paris-Saclay, France (2016).

[45] Z. Bern, L.J. Dixon, M. Perelstein and J.S. Rozowsky, Multileg one loop gravity amplitudes from gauge theory, Nucl. Phys. B 546 (1999) 423 [hep-th/9811140] [inSPIRE]. 